\title{
On the energy of domain walls in ferromagnetism
}

\author{
ROGER MOSER \\ Department of Mathematical Sciences, University of Bath, Bath BA2 7AY, UK \\ E-mail: r.moser@maths.bath.ac.uk
}

[Received 13 March 2008 and in revised form 6 January 2009]

\begin{abstract}
In the theory of micromagnetics, the magnetization of a ferromagnetic sample has an energy that is the sum of four components. We study the asymptotic behaviour of this functional when a parameter (the so-called exchange length) tends to 0 . The interaction of two of the components of the energy permits the use of well-known methods from the theory of phase transitions. In the limit this gives rise to a division of the sample into domains of constant magnetization, separated by domain walls. We examine the contribution of a third energy (the energy of the stray field) to the limiting problem. In particular, we derive an estimate for the energy density on the domain walls.
\end{abstract}

\section{Introduction and motivation}

Suppose that a bounded, open set $\Omega \subset \mathbb{R}^{3}$ represents the shape of a sample of ferromagnetic material. This ferromagnet has a magnetization described by a vector field $m: \Omega \rightarrow \mathbb{R}^{3}$. Below the Curie temperature, the magnetization is saturated, which means that $m$ is of constant length. After a renormalization we have $|\boldsymbol{m}|=1$ in $\Omega$, thus $\boldsymbol{m}$ can be interpreted as a map from $\Omega$ into the unit 2-sphere $S^{2}$. In the theory of micromagnetics, an energy is associated to a given magnetization, consisting of several components.

- The exchange energy is derived from the Heisenberg interaction of neighbouring spins in the underlying atomic model. It is given by

$$
\epsilon^{2} \int_{\Omega}|\nabla \boldsymbol{m}|^{2} \mathrm{~d} \boldsymbol{x}
$$

where $\epsilon$ is a material constant, called the exchange length.

- The crystalline anisotropy of the material favours certain directions of the magnetization. This is modelled by a smooth function $\Psi: S^{2} \rightarrow \mathbb{R}$ with several minima that correspond to the preferred directions. The anisotropy energy is then

$$
\int_{\Omega} \Psi(\boldsymbol{m}) \mathrm{d} \boldsymbol{x}
$$

- The magnetization induces a stray field $\boldsymbol{h}$ on $\mathbb{R}^{3}$. By the static Maxwell equations, this is a gradient field, that is, we have a potential function $u$ on $\mathbb{R}^{3}$ with $\boldsymbol{h}=-\nabla u$. Extending $\boldsymbol{m}$ by 0 outside of $\Omega$, we further have

$$
\Delta u=\operatorname{div} \boldsymbol{m} \text { in } \mathbb{R}^{3} .
$$

If we assume that $u$ belongs to the Sobolev space $H^{1}\left(\mathbb{R}^{3}\right)$, then this equation determines $u$ uniquely. The contribution of the stray field to the micromagnetic energy is

$$
\int_{\mathbb{R}^{3}}|\nabla u|^{2} \mathrm{~d} \boldsymbol{x} .
$$

This is called the magnetostatic energy. 
- If there is an external magnetic field $\boldsymbol{H}$, then it gives rise to the energy

$$
-2 \int_{\Omega} \boldsymbol{H} \cdot \boldsymbol{m} \mathrm{d} \boldsymbol{x} .
$$

The total micromagnetic energy is the sum of all four terms, i.e.,

$$
\mathcal{E}(\boldsymbol{m})=\int_{\Omega}\left(\epsilon^{2}|\nabla \boldsymbol{m}|^{2}+\Psi(\boldsymbol{m})-2 \boldsymbol{H} \cdot \boldsymbol{m}\right) \mathrm{d} \boldsymbol{x}+\int_{\mathbb{R}^{3}}|\boldsymbol{\nabla} u|^{2} \mathrm{~d} \boldsymbol{x} .
$$

More information about its derivation can be found, e.g., in books by Hubert and Schäfer [10] and Bertotti [5].

If the external field is constant, then its energy and the anisotropy energy have a similar structure. Setting $\Phi(\boldsymbol{m})=\Psi(\boldsymbol{m})-2 \boldsymbol{H} \cdot \boldsymbol{m}$, we can combine them. Since adding a constant will not change the qualitative behaviour of the functional, we normally assume that

$$
\min _{S^{2}} \Phi=0 .
$$

For the purpose of this paper, it is further convenient to divide the energy by $\epsilon$. Thus we obtain the functional

$$
E_{\epsilon}(\boldsymbol{m})=\int_{\Omega}\left(\epsilon|\nabla \boldsymbol{m}|^{2}+\frac{1}{\epsilon} \Phi(\boldsymbol{m})\right) \mathrm{d} \boldsymbol{x}+\frac{1}{\epsilon} \int_{\mathbb{R}^{3}}|\nabla u|^{2} \mathrm{~d} \boldsymbol{x},
$$

and it is natural to study it on the nonlinear Sobolev space

$$
H^{1}\left(\Omega, S^{2}\right)=\left\{\boldsymbol{m} \in H^{1}\left(\Omega, \mathbb{R}^{3}\right):|\boldsymbol{m}|=1 \text { almost everywhere }\right\} .
$$

The exchange length $\epsilon$ is often very small relative to the size of the sample, thus it is reasonable to consider the limit $\epsilon \searrow 0$. The aim of this paper is to study the asymptotic behaviour of $E_{\epsilon}$ for this limit. In order to explain the structure of the expected limiting energy, we first describe some known arguments for simplified versions of the problem.

At first, we neglect the magnetostatic energy. We assume that we have a family of magnetizations $m_{\epsilon}$ such that

$$
\limsup _{\epsilon \searrow 0} \int_{\Omega}\left(\epsilon\left|\nabla \boldsymbol{m}_{\epsilon}\right|^{2}+\frac{1}{\epsilon} \Phi\left(\boldsymbol{m}_{\epsilon}\right)\right) \mathrm{d} \boldsymbol{x}<\infty .
$$

If $\Phi$ has finitely many zeroes, say at $a_{1}, \ldots, \boldsymbol{a}_{n} \in S^{2}$, then we can use methods from the theory of phase transitions (cf. Modica and Mortola [13], Modica [12], Sternberg [17], Fonseca and Tartar [9], Baldo [4]). In the context studied here, they have first been used by Anzellotti, Baldo, and Visintin [3]. The main idea is to equip the sphere $S^{2}$ with a new metric depending on $\Phi$. If $g$ denotes the standard Riemannian metric on $S^{2}$, then we define

$$
g_{\Phi}=4 \Phi g .
$$

This is a Riemannian metric on $S^{2} \backslash\left\{\boldsymbol{a}_{1}, \ldots, \boldsymbol{a}_{n}\right\}$. At the points $\boldsymbol{a}_{1}, \ldots, \boldsymbol{a}_{n}$ it becomes degenerate, but it still induces a metric $\delta_{\Phi}$ on the whole of $S^{2}$. Namely, for $\boldsymbol{y}, \boldsymbol{z} \in S^{2}$, we consider the space $\Gamma(\boldsymbol{y}, \boldsymbol{z})$ of Lipschitz continuous curves in $S^{2}$ connecting them, i.e.,

$$
\Gamma(\boldsymbol{y}, \boldsymbol{z})=\left\{\gamma \in C^{0,1}\left([0,1], S^{2}\right): \gamma(0)=\boldsymbol{y} \text { and } \gamma(1)=\boldsymbol{z}\right\},
$$


and we define

$$
\delta_{\Phi}(\boldsymbol{y}, \boldsymbol{z})=\inf _{\boldsymbol{\gamma} \in \Gamma(\boldsymbol{y}, \boldsymbol{z})} \int_{0}^{1} \sqrt{g_{\Phi}\left(\gamma^{\prime}, \gamma^{\prime}\right)} \mathrm{d} t=2 \inf _{\boldsymbol{\gamma} \in \Gamma(\boldsymbol{y}, \boldsymbol{z})} \int_{0}^{1} \sqrt{\Phi(\gamma)}\left|\gamma^{\prime}\right| \mathrm{d} t .
$$

For any $z \in S^{2}$, the function

$$
\phi(\boldsymbol{y})=\delta_{\Phi}(\boldsymbol{y}, \boldsymbol{z})
$$

is Lipschitz continuous. Writing grad for the gradient on the sphere $S^{2}$, we have

$$
|\operatorname{grad} \phi(y)| \leqslant 2 \sqrt{\Phi(y)}
$$

almost everywhere on $S^{2}$. Hence for $\boldsymbol{m} \in H^{1}\left(\Omega, S^{2}\right)$ we obtain

$$
|\nabla \phi(\boldsymbol{m})| \leqslant 2|\nabla \boldsymbol{m}| \sqrt{\Phi(\boldsymbol{m})} \leqslant \epsilon|\nabla \boldsymbol{m}|^{2}+\frac{1}{\epsilon} \Phi(\boldsymbol{m})
$$

by Young's inequality. We conclude that the functions $\phi\left(\boldsymbol{m}_{\epsilon}\right)$ are uniformly bounded in the space of functions of bounded variation $\operatorname{BV}(\Omega)$. Since this holds for any fixed $z \in S^{2}$, it is then clear that $\Omega$ is divided into domains $\Omega_{1}, \ldots, \Omega_{n}$ such that for a suitable sequence $\epsilon_{k} \searrow 0$, we have $\boldsymbol{m}_{\epsilon_{k}}(\boldsymbol{x}) \rightarrow \boldsymbol{a}_{i}$ almost everywhere in $\Omega_{i}$ for $i=1, \ldots, n$.

When we require that even

$$
\limsup _{\epsilon \searrow 0} E_{\epsilon}\left(\boldsymbol{m}_{\epsilon}\right)<\infty,
$$

then we can of course use the same arguments. In addition, it follows that the map $\boldsymbol{m}: \Omega \rightarrow S^{2}$ with $\boldsymbol{m}(\boldsymbol{x})=\boldsymbol{a}_{i}$ for $\boldsymbol{x} \in \Omega_{i}, i=1, \ldots, n$, must be divergence free in the sense of distributions. That is, $\boldsymbol{m}$ is tangential at the boundary $\partial \Omega$, and if the domains $\Omega_{i}$ and $\Omega_{j}$ have a common boundary, then its tangent spaces must contain $\boldsymbol{a}_{i}-\boldsymbol{a}_{j}$. As $\boldsymbol{m}$ takes only finitely many values, the first condition is inconsistent with most shapes of $\Omega$. It is possible to choose $\Omega$ such that this problem does not arise, but since we are interested in the behaviour of the magnetization in the interior of $\Omega$, we prefer to avoid this issue altogether by neglecting the contribution of $\partial \Omega$ to the stray field. This may be justified if the boundary is very far away from the region that we study. (Incidentally, the behaviour of the magnetization near the boundary shows interesting phenomena that are related to this observation; cf. Choksi and Kohn [6] or Choksi, Kohn, and Otto [7].) The condition on the interfaces between $\Omega_{i}$ and $\Omega_{j}$, on the other hand, is crucial for the analysis in this paper.

The behaviour described above is consistent with the experimental observation of domains with nearly constant magnetizations, separated by thin layers where it changes rapidly. In simplified models, the transition layers are often replaced by sharp interfaces, called domain walls. Such an approach requires information about the energy concentrated at the domain walls, and this is where we turn our attention next. Again we first examine a simplified situation; we will see later that it is in fact oversimplified, but nevertheless it demonstrates some of the questions that arise in this context.

We consider the profile of a transition between two minima of the function $\Phi$, say $\boldsymbol{a}_{1}$ and $\boldsymbol{a}_{2}$, across a domain wall parallel to $\mathbb{R}^{2} \times\{0\}$. We assume that the transition is described by a vector field $\boldsymbol{m}: \mathbb{R}^{3} \rightarrow S^{2}$ that depends only on the third variable. That is, we have

$$
\boldsymbol{m}\left(x_{1}, x_{2}, x_{3}\right)=\boldsymbol{n}\left(x_{3}\right)
$$

for a curve $n: \mathbb{R} \rightarrow S^{2}$ with

$$
\lim _{x_{3} \rightarrow-\infty} \boldsymbol{n}\left(x_{3}\right)=\boldsymbol{a}_{1} \quad \text { and } \quad \lim _{x_{3} \rightarrow+\infty} \boldsymbol{n}\left(x_{3}\right)=\boldsymbol{a}_{2}
$$


Such a situation might occur as a limiting case if the maps $\boldsymbol{m}_{\epsilon}$ in the previous considerations are rescaled about a point on the domain wall. If we neglect the magnetostatic energy again, then this gives rise to the functional

$$
E^{*}(\boldsymbol{n})=\int_{-\infty}^{\infty}\left(\left|\boldsymbol{n}^{\prime}\right|^{2}+\Phi(\boldsymbol{n})\right) \mathrm{d} x_{3} .
$$

(The parameter $\epsilon$ is renormalized to 1 by the rescaling mentioned above.) Using the same arguments as before, we see that

$$
E^{*}(\boldsymbol{n}) \geqslant \delta_{\Phi}\left(\boldsymbol{a}_{1}, \boldsymbol{a}_{2}\right) .
$$

Moreover, the transition profile with the least energy follows a shortest geodesic for the Riemannian metric $g_{\Phi}$ connecting $\boldsymbol{a}_{1}$ and $\boldsymbol{a}_{2}$.

Now we take the magnetostatic energy into account again. If we assume that $u: \mathbb{R}^{3} \rightarrow \mathbb{R}$ is a function of the form

$$
u\left(x_{1}, x_{2}, x_{3}\right)=v\left(x_{3}\right),
$$

then condition (1) becomes

$$
v^{\prime \prime}=n_{3}^{\prime} .
$$

Thus $v^{\prime}$ and $n_{3}$ differ by a constant, say $n_{3}-v^{\prime}=a$. If we insist that

$$
\int_{-\infty}^{\infty}\left(v^{\prime}\right)^{2} \mathrm{~d} x_{3}<\infty
$$

then $a$ must satisfy

$$
a=\lim _{x_{3} \rightarrow-\infty} n_{3}\left(x_{3}\right)=\lim _{x_{3} \rightarrow+\infty} n_{3}\left(x_{3}\right) .
$$

In particular, we have again the condition that $\boldsymbol{a}_{1}-\boldsymbol{a}_{2}$ is tangential to the domain wall. Moreover, we obtain the new functional

$$
E^{\#}(\boldsymbol{n})=\int_{-\infty}^{\infty}\left(\left|\boldsymbol{n}^{\prime}\right|^{2}+\Phi(\boldsymbol{n})+\left(n_{3}-a\right)^{2}\right) \mathrm{d} x_{3} .
$$

Setting $F(\boldsymbol{y})=\left(y_{3}-a\right)^{2}$, we conclude that

$$
E^{\#}(\boldsymbol{n}) \geqslant \delta_{\Phi+F}\left(\boldsymbol{a}_{1}, \boldsymbol{a}_{2}\right) .
$$

If $\boldsymbol{a}_{1}$ and $\boldsymbol{a}_{2}$ are connected by a shortest geodesic for $g_{\Phi}$ that is entirely in the plane $\mathbb{R}^{2} \times\{a\}$, then

$$
\delta_{\Phi+F}\left(\boldsymbol{a}_{1}, \boldsymbol{a}_{2}\right)=\delta_{\Phi}\left(\boldsymbol{a}_{1}, \boldsymbol{a}_{2}\right) .
$$

The optimal transition profile then follows exactly such a geodesic. The corresponding $\boldsymbol{m}$ has a constant third component, thus it is divergence free, and there is no magnetostatic energy. A domain wall with such a transition path is called a Bloch wall. In any other case, we have

$$
\delta_{\Phi+F}\left(\boldsymbol{a}_{1}, \boldsymbol{a}_{2}\right)>\delta_{\Phi}\left(\boldsymbol{a}_{1}, \boldsymbol{a}_{2}\right)
$$

That is, the magnetostatic energy contributes to the wall energy. The optimal transition path is now a geodesic for $g_{\Phi+F}$. More details on an analysis of this model can be found in Section 3.6.1(F) of [10]. 
While such a reasoning may give some insight, it requires strong assumptions on the structure of the domain wall and the magnetization vector field. Particularly important is the assumption that the transition between two minima of $\Phi$ is described by a magnetization that depends only on one variable. In this paper we derive rigorous estimates for the limiting domain wall energy without using such assumptions. The results are stated in the next section, but we can summarize them now with the following conclusions.

1. The magnetostatic energy contributes to the limiting energy. At least a part of this contribution is given by the integral of a certain density function over the domain walls. In contrast to the nonlocal expression $|\nabla u|^{2}$, this function depends only on the local behaviour of the limiting magnetization.

2. The limiting energy density depends on the orientation of the domain walls. The part coming from the magnetostatic energy vanishes only where the transition path of a Bloch wall coincides with the optimal transition path for the anisotropy energy.

3. The domain wall energy may be smaller, however, than the above analysis suggests. In certain cases the development of fine structures near the domain walls can decrease the energy.

\section{Statement of the results}

In order to simplify the presentation of our analysis, we choose a specific function $\Phi$ for the rest of the paper. This function corresponds to a uniaxial ferromagnetic material in an external magnetic field perpendicular to the 'easy axis'. More precisely, if we choose

$$
\Psi(\boldsymbol{y})=y_{2}^{2}+y_{3}^{2}, \quad \boldsymbol{y} \in S^{2},
$$

then the anisotropy energy favours a magnetization parallel to $\mathbb{R} \times\{(0,0)\}$. The minima of $\Psi$ are shifted to an asymmetric position by the influence of an external field of the form

$$
\boldsymbol{H}=\left(0, H_{2}, H_{3}\right)
$$

with $0<H_{2}^{2}+H_{3}^{2}<1$. We consider the function

$$
\Phi(\boldsymbol{y})=\Psi(\boldsymbol{y})-2 \boldsymbol{H} \cdot \boldsymbol{y}+|\boldsymbol{H}|^{2}=\left(y_{2}-H_{2}\right)^{2}+\left(y_{3}-H_{3}\right)^{2}
$$

(the term $|\boldsymbol{H}|^{2}$ is added to make it nonnegative) with minima at

$$
\boldsymbol{a}_{+}=\left(\sqrt{1-H_{2}^{2}-H_{3}^{2}}, H_{2}, H_{3}\right) \quad \text { and } \quad \boldsymbol{a}_{-}=\left(-\sqrt{1-H_{2}^{2}-H_{3}^{2}}, H_{2}, H_{3}\right) .
$$

In this situation we expect domain walls with normal vectors in the circle

$$
N=\left\{\boldsymbol{\nu} \in S^{2}: \boldsymbol{\nu} \perp \boldsymbol{a}_{+}-\boldsymbol{a}_{-}\right\} .
$$

Since we will often compare the distances between $\boldsymbol{a}_{+}$and $\boldsymbol{a}_{-}$for different metrics on $S^{2}$, we introduce an abbreviation. Suppose that $\Xi: S^{2} \rightarrow[0, \infty)$ is a smooth function with $\Xi^{-1}(\{0\})=$ $\left\{\boldsymbol{a}_{+}, \boldsymbol{a}_{-}\right\}$. Then we set

$$
\mathcal{D}(\Xi)=\delta_{\Xi}\left(\boldsymbol{a}_{+}, \boldsymbol{a}_{-}\right),
$$

where $\delta_{\Xi}$ is defined as in the previous section. For example, we can compute

$$
\mathcal{D}(\Phi)=4\left(\sqrt{1-|\boldsymbol{H}|^{2}}-|\boldsymbol{H}| \arccos |\boldsymbol{H}|\right) .
$$


As mentioned previously, we ignore the effect of the boundary $\partial \Omega$ on the micromagnetic energy. In fact, instead of using equation (1), we only require that $u$ satisfies $\Delta u=\operatorname{div} \boldsymbol{m}$ in $\Omega$ henceforth. We then consider the integral

$$
\int_{\Omega}\left(\epsilon|\nabla \boldsymbol{m}|^{2}+\frac{1}{\epsilon}\left(\Phi(\boldsymbol{m})+|\nabla u|^{2}\right)\right) \mathrm{d} \boldsymbol{x} .
$$

Rather than a functional of $\boldsymbol{m}$ alone, this is now a functional of $\boldsymbol{m}$ and the trace of $u$ on $\partial \Omega$ (provided that $\partial \Omega$ is sufficiently smooth to speak of a trace).

Before we can state the main result, we need to introduce a few notions from geometric measure theory. First, we write $\mathcal{H}^{2}$ for the 2-dimensional Hausdorff measure. We have already used the space $\operatorname{BV}(\Omega)$, which consists of all functions $f \in L^{1}(\Omega)$ such that the distributional derivative is represented by a vector valued Radon measure $D f$ on $\Omega$. This means in particular that $D f$ can be interpreted as and element of the dual space of $C_{0}^{0}\left(\Omega, \mathbb{R}^{3}\right)$. The Radon measure $|\boldsymbol{D} f|$ is defined by

$$
|\boldsymbol{D} f|(U)=\sup \left\{\int_{\Omega} f \operatorname{div} \xi \mathrm{d} \boldsymbol{x}: \xi \in C_{0}^{1}\left(U, \mathbb{R}^{3}\right) \text { with } \sup _{U}|\xi| \leqslant 1\right\}
$$

for any open set $U \subset \Omega$. A subset $A \subset \Omega$ has finite perimeter in $\Omega$ if its characteristic function $\chi_{A}$ belongs to $\operatorname{BV}(\Omega)$. In this case, the reduced boundary $\mathcal{F} A$ of $A$ is the set of all points $x \in$ $\operatorname{supp}\left|\boldsymbol{D} \chi_{A}\right| \cap \Omega$ such that the limit

$$
\boldsymbol{\nu}_{A}(\boldsymbol{x})=\lim _{r \searrow 0} \frac{\boldsymbol{D} \chi_{A}\left(B_{r}(\boldsymbol{x})\right)}{\left|\boldsymbol{D} \chi_{A}\right|\left(B_{r}(\boldsymbol{x})\right)}
$$

exists and belongs to $S^{2}$. The measure $\left|\boldsymbol{D} \chi_{A}\right|$ is then the restriction of $\mathcal{H}^{2}$ to $\mathcal{F} A$, i.e.,

$$
\left|D \chi_{A}\right|=\mathcal{H}^{2}\llcorner\mathcal{F} A \text {. }
$$

The set $\mathcal{F} A$ is the measure-theoretically relevant part of the boundary of $A$ and $\nu_{A}$ is its normal vector (pointing into $A$ ). More details can be found in a book by Ambrosio, Fusco, and Pallara [2].

THEOREM 2.1 There exists a function $W: N \rightarrow[\mathcal{D}(\Phi), \infty)$ with

$$
W^{-1}(\{\mathcal{D}(\Phi)\})=\{\boldsymbol{\nu} \in N: \boldsymbol{\nu} \perp \boldsymbol{H}\}
$$

such that the following holds. For $\epsilon \in(0,1]$, suppose that $\boldsymbol{m}_{\epsilon} \in H^{1}\left(\Omega, S^{2}\right)$ and $u_{\epsilon} \in H^{2}(\Omega)$ satisfy $\Delta u_{\epsilon}=\operatorname{div} \boldsymbol{m}_{\epsilon}$ in $\Omega$. If

$$
\liminf _{\epsilon \searrow 0} \int_{\Omega}\left(\epsilon\left|\nabla \boldsymbol{m}_{\epsilon}\right|^{2}+\frac{1}{\epsilon}\left(\Phi\left(\boldsymbol{m}_{\epsilon}\right)+\left|\nabla u_{\epsilon}\right|^{2}\right)\right) \mathrm{d} \boldsymbol{x}<\infty,
$$

then there exist a sequence $\epsilon_{k} \searrow 0$ and a set $\Omega_{+}$of finite perimeter in $\Omega$ such that $\boldsymbol{m}_{\epsilon_{k}}(\boldsymbol{x}) \rightarrow \boldsymbol{a}_{+}$for almost all $\boldsymbol{x} \in \Omega_{+}$and $\boldsymbol{m}_{\epsilon_{k}}(\boldsymbol{x}) \rightarrow \boldsymbol{a}_{-}$for almost all $\boldsymbol{x} \in \Omega \backslash \Omega_{+}$. For $\mathcal{H}^{2}$-almost every $\boldsymbol{x} \in \mathcal{F} \Omega_{+}$, the vector $\nu_{\Omega_{+}}(\boldsymbol{x})$ belongs to $N$. Moreover,

$$
\int_{\mathcal{F} \Omega_{+}} \eta W\left(\nu_{\Omega_{+}}\right) \mathrm{d} \mathcal{H}^{2} \leqslant \liminf _{k \rightarrow \infty} \int_{\Omega} \eta\left(\epsilon_{k}\left|\nabla \boldsymbol{m}_{\epsilon_{k}}\right|^{2}+\frac{1}{\epsilon_{k}}\left(\Phi\left(m_{\epsilon_{k}}\right)+\left|\nabla u_{\epsilon_{k}}\right|^{2}\right)\right) \mathrm{d} \boldsymbol{x}
$$

for every nonnegative $\eta \in C_{0}^{0}(\Omega)$. 
The convergence of a sequence as described in this theorem, and some of the other statements, are consequences of the known arguments seen in Section 1 . The main new aspect is inequality [2), which gives an estimate for the energy density on the domain walls. Without the magnetostatic energy, we would have a similar inequality with $\mathcal{D}(\Phi)$ instead of $W\left(\boldsymbol{\nu}_{\Omega_{+}}\right)$; therefore, the magnetostatic energy contributes to the domain wall energy at least with the density $W\left(\boldsymbol{\nu}_{\Omega_{+}}(\boldsymbol{x})\right)-$ $\mathcal{D}(\Phi)$ at a point $\boldsymbol{x} \in \mathcal{F} \Omega_{+}$. This difference is positive unless $\boldsymbol{\nu}_{\Omega_{+}}(\boldsymbol{x})$ is one of the two vectors in $N$ perpendicular to $\boldsymbol{H}$. In particular, we conclude that the magnetostatic energy is not negligible in the limit, unless the domain wall $\mathcal{F} \Omega_{+}$is contained in a collection of planes parallel to the plane spanned by $\boldsymbol{a}_{+}-\boldsymbol{a}_{-}$and $\boldsymbol{H}$. The theorem does not give any further information about the function $W$, and although more explicit estimates could be obtained from the proof in principle, they would probably be too complicated for practical purposes and certainly far from optimal. Further work is clearly needed in this context.

It is not difficult to see that similar statements as in Theorem 2.1 hold if $\Phi$ is replaced by a function with more than two (but finitely many) minima. This situation occurs, e.g., for ferromagnetic materials with cubic anisotropy. In this case, the results are also relevant in the absence of an external field. For the uniaxial model with no external field, on the other hand, the walls with the least energy are the Bloch walls, for which there is no contribution from the magnetostatic energy in the limit.

In view of the one-dimensional analysis in Section 1, it is tempting to conjecture that $W(\boldsymbol{\nu})=$ $\mathcal{D}\left(\Phi+F^{\nu}\right)$ for the function $F^{\boldsymbol{\nu}}(\boldsymbol{y})=\left(\boldsymbol{\nu} \cdot\left(\boldsymbol{y}-\boldsymbol{a}_{+}\right)\right)^{2}$. But in fact this is not true, at least not if $|\boldsymbol{H}|$ is small enough.

Proposition 2.1 Suppose that $\Omega=(-1,1)^{3}$ and $H_{2}=0$, and let $\nu=(0,0,1)$. There exists a number $\beta \in(0,1)$ such that whenever $0<H_{3} \leqslant \beta$, there exist

- a sequence $\epsilon_{k} \searrow 0$,

- a sequence of maps $\boldsymbol{m}_{k} \in H^{1}\left(\Omega, S^{2}\right)$ with $\boldsymbol{m}_{k} \rightarrow \boldsymbol{a}_{-}$almost everywhere in $(-1,1)^{2} \times(-1,0)$

and $\boldsymbol{m}_{k} \rightarrow \boldsymbol{a}_{+}$almost everywhere in $(-1,1)^{2} \times(0,1)$, and

- a sequence of functions $u_{k} \in H^{2}(\Omega)$ with $\Delta u_{k}=\operatorname{div} \boldsymbol{m}_{k}$ in $\Omega$,

such that

$$
\limsup _{k \rightarrow \infty} \int_{\Omega}\left(\epsilon_{k}\left|\nabla \boldsymbol{m}_{k}\right|^{2}+\frac{1}{\epsilon_{k}}\left(\Phi\left(\boldsymbol{m}_{k}\right)+\left|\nabla u_{k}\right|^{2}\right)\right) \mathrm{d} \boldsymbol{x}<4 \mathcal{D}\left(\Phi+F^{\boldsymbol{\nu}}\right) .
$$

This statement is restricted to a special situation in order to keep the construction in its proof as simple as possible. But similar arguments work also more generally, and a further examination would give an explicit upper bound for $W$. The construction that we use mimics a phenomenon observed in experiments: under certain conditions, a domain wall perpendicular to the external field tends to develop zigzag patterns (cf. Section 3.6.2(B) in [10] for a further discussion).

It is interesting to compare the problem studied here with the asymptotic analysis of Rivière and Serfaty [15, 16]. In these papers, a two-dimensional model is studied, where the magnetization is a map from a domain in $\mathbb{R}^{2}$ into the unit circle $S^{1}$. The energy functional consists of two terms, corresponding to the exchange energy and the magnetostatic energy, and there is no anisotropy term. In three dimensions, we could have Bloch walls with no limiting energy in the absence of an anisotropy energy. In two dimensions this is impossible, because the constraint $|\boldsymbol{m}|=1$ is incompatible with Bloch walls. Accordingly, Rivière and Serfaty find a non-vanishing limiting energy. A different, but related model is studied by Alouges, Rivière, and Serfaty [1]. 
The above observations suggest that the three-dimensional theory might be closer to a twodimensional model that replaces the constraint $|\boldsymbol{m}|=1$ with a penalizing term in the functional, such as the integral of $\epsilon^{-1}\left(1-|\boldsymbol{m}|^{2}\right)^{2}$. A problem of this kind has been studied by Jabin, Otto, and Perthame [11] and by De Lellis and Otto [8], albeit in a different context. The methods used, and the methods of the aforementioned papers [15, 16, 1] as well, depend on the fact that the domain is two-dimensional. It is not clear how to generalize them to higher dimensions.

\section{Energy estimates}

In this section we derive the main tools for the proof of inequality (2). We work mostly in cubes and cuboids here. For $\boldsymbol{x} \in \mathbb{R}^{3}$ and $r>0$, we define

$$
Q_{r}(\boldsymbol{x})=\left(x_{1}-r, x_{1}+r\right) \times\left(x_{2}-r, x_{2}+r\right) \times\left(x_{3}-r, x_{3}+r\right) .
$$

Because we often consider two-dimensional slices, we use the notation $\boldsymbol{x}=\left(\boldsymbol{x}^{\prime}, x_{3}\right)=\left(x_{1}, x_{2}, x_{3}\right)$ for a generic point in $\mathbb{R}^{3}$. Similarly, we write $\nabla^{\prime}=\left(\partial / \partial x_{1}, \partial / \partial x_{2}\right)$, so that $\boldsymbol{\nabla}=\left(\boldsymbol{\nabla}^{\prime}, \partial / \partial x_{3}\right)$. For $\boldsymbol{x}^{\prime} \in \mathbb{R}^{2}$ and $r>0$, we define the square

$$
Q_{r}^{\prime}\left(\boldsymbol{x}^{\prime}\right)=\left(x_{1}-r, x_{1}+r\right) \times\left(x_{2}-r, x_{2}+r\right) .
$$

An open ball in $\mathbb{R}^{3}$ with radius $r$ and centre $\boldsymbol{x}$ is denoted by $B_{r}(\boldsymbol{x})$.

We fix a number $\tau>0$ (and it will stay fixed throughout this section). On $S^{2}$, we then define the function

$$
G_{\tau}(\boldsymbol{y})=\left(\max \left\{\left|y_{3}^{2}-H_{3}\right|-\tau, 0\right\}\right)^{2} .
$$

The following is the key ingredient in the proof of Theorem 2.1

Proposition 3.1 There exist two constants $c$ and $\epsilon_{0}>0$, dependent only on $\tau$, with the following property. Suppose that $\epsilon \in\left(0, \epsilon_{0}\right]$. Let $\boldsymbol{m} \in H^{1}\left(Q_{1}(\mathbf{0}), S^{2}\right)$ and $u \in H^{2}\left(Q_{1}(\mathbf{0})\right)$ such that $\Delta u=$ $\operatorname{div} \boldsymbol{m}$ in $Q_{1}(\mathbf{0})$. Then for any $\theta \in(0,1]$,

$$
\begin{aligned}
\int_{Q_{1}(\mathbf{0})}\left(\epsilon|\nabla \boldsymbol{m}|^{2}+\frac{1}{\epsilon}\left(\Phi(\boldsymbol{m})+|\nabla u|^{2}\right)\right) \mathrm{d} \boldsymbol{x} & \\
& \geqslant \int_{Q_{1}^{\prime}(\mathbf{0})} \delta_{\left(1-c \theta^{2}\right)\left(\Phi+\theta G_{\tau}\right)}\left(\boldsymbol{m}\left(\boldsymbol{x}^{\prime},-1\right), \boldsymbol{m}\left(\boldsymbol{x}^{\prime}, 1\right)\right) \mathrm{d} \boldsymbol{x}^{\prime} .
\end{aligned}
$$

Before we can prove this result, we need some preparation. First we introduce an auxiliary variational problem. For $L \geqslant 1$, let $\Omega_{L}=Q_{1}^{\prime}(\mathbf{0}) \times(-L, L)$. If $\boldsymbol{m}^{*} \in H^{1}\left(\Omega_{L}, S^{2}\right)$ and $u^{*} \in$ $H^{2}\left(\Omega_{L}\right)$ are given, then we consider the space

$$
\mathcal{A}_{L}\left(\boldsymbol{m}^{*}\right)=\left\{\boldsymbol{m} \in H^{1}\left(\Omega_{L}, S^{2}\right): \boldsymbol{m}=\boldsymbol{m}^{*} \text { on } Q_{1}^{\prime}(\mathbf{0}) \times\{-L, L\}\right\} .
$$

For $\boldsymbol{m} \in \mathcal{A}_{L}\left(\boldsymbol{m}^{*}\right)$, let $u \in H^{2}\left(\Omega_{L}\right)$ be the unique solution of the boundary value problem

$$
\begin{aligned}
\Delta u & =\operatorname{div} \boldsymbol{m} & & \text { in } \Omega_{L}, \\
\nu_{\Omega_{L}} \cdot(\nabla u-\boldsymbol{m}) & =\boldsymbol{\nu}_{\Omega_{L}} \cdot\left(\nabla u^{*}-\boldsymbol{m}^{*}\right) & & \text { on } \partial \Omega_{L}
\end{aligned}
$$

with

$$
\int_{\Omega_{L}} u \mathrm{~d} \boldsymbol{x}=\int_{\Omega_{L}} u^{*} \mathrm{~d} \boldsymbol{x} .
$$


Then we define

$$
\mathcal{J}_{L}\left(\boldsymbol{m} ; \boldsymbol{m}^{*}, u^{*}\right)=\int_{\Omega_{L}}\left(|\nabla \boldsymbol{m}|^{2}+\Phi(\boldsymbol{m})+|\nabla u|^{2}\right) \mathrm{d} \boldsymbol{x} .
$$

A minimizer of $\mathcal{J}_{L}\left(\cdot ; \boldsymbol{m}^{*}, u^{*}\right)$ in $\mathcal{A}_{L}\left(\boldsymbol{m}^{*}\right)$ can always be constructed with the direct method.

LEMMA 3.1 There exists a constant $\Lambda>0$, dependent only on $\tau$, with the following property. For $L \geqslant 2$, let $\boldsymbol{m}^{*} \in H^{1}\left(\Omega_{L}, S^{2}\right)$ and $u^{*} \in H^{2}\left(\Omega_{L}\right)$. Suppose that $\boldsymbol{m} \in H^{1}\left(\Omega_{L}, S^{2}\right)$ is a minimizer of $\mathcal{J}_{L}\left(\cdot ; \boldsymbol{m}^{*}, u^{*}\right)$ in $\mathcal{A}_{L}\left(\boldsymbol{m}^{*}\right)$ and $u \in H^{2}(\Omega)$ is the corresponding solution of 3 . Then for any $s \in[2-L, L-2]$, either $\boldsymbol{m} \in C^{0}\left(\overline{Q_{1}(\mathbf{0}, s)}, S^{2}\right)$ with

$$
\underset{Q_{1}(\mathbf{0}, s)}{\operatorname{osc}} \boldsymbol{m} \leqslant \tau / 2
$$

or

$$
\int_{Q_{1}^{\prime}(\mathbf{0}) \times(s-2, s+2)}\left(|\boldsymbol{\nabla} \boldsymbol{m}|^{2}+\Phi(\boldsymbol{m})+|\nabla u|^{2}\right) \mathrm{d} \boldsymbol{x} \geqslant \Lambda .
$$

Proof. We use well-known methods from the theory of harmonic maps, combined with results that are proved elsewhere. Nevertheless, we need to give a few details of the arguments, since the nonlocal term in the functional $\mathcal{J}_{L}\left(\cdot ; \boldsymbol{m}^{*}, u^{*}\right)$ requires some special attention.

Let $s \in[2-L, L-2]$ and set

$$
\lambda=\int_{Q_{1}^{\prime}(\mathbf{0}) \times(s-2, s+2)}\left(|\nabla \boldsymbol{m}|^{2}+\Phi(\boldsymbol{m})+|\nabla u|^{2}\right) \mathrm{d} \boldsymbol{x} .
$$

We may assume that $\lambda \leqslant 1$. Let $\kappa>0$ be a constant, the value of which will be determined later. In the first step, we want to show that there exists a constant $C_{1}=C_{1}(\kappa)$ such that

$$
\frac{1}{r} \int_{B_{r}\left(\boldsymbol{x}_{0}\right) \cap \Omega_{L}}|\nabla \boldsymbol{m}|^{2} \mathrm{~d} \boldsymbol{x} \leqslant C_{1} \lambda+\kappa
$$

for all $\boldsymbol{x}_{0} \in Q_{1}^{\prime}(\mathbf{0}) \times(s-3 / 2, s+3 / 2)$ and $r \in(0,1 / 2]$.

To this end, assume first that $B_{R}\left(\boldsymbol{x}_{0}\right) \subset \Omega_{L}$. For $\rho \in(0, R]$ consider the map

$$
\tilde{\boldsymbol{m}}(\boldsymbol{x})= \begin{cases}\boldsymbol{m}(\boldsymbol{x}) & \text { if }\left|\boldsymbol{x}-\boldsymbol{x}_{0}\right| \geqslant \rho, \\ \boldsymbol{m}\left(\boldsymbol{x}_{0}+\rho \frac{\boldsymbol{x}-\boldsymbol{x}_{0}}{\left|\boldsymbol{x}-\boldsymbol{x}_{0}\right|}\right) & \text { if }\left|\boldsymbol{x}-\boldsymbol{x}_{0}\right|<\rho .\end{cases}
$$

It is readily checked that

$$
\int_{B_{\rho}\left(\boldsymbol{x}_{0}\right)}\left(|\nabla \tilde{\boldsymbol{m}}|^{2}+\Phi(\tilde{\boldsymbol{m}})\right) \mathrm{d} \boldsymbol{x} \leqslant \rho \int_{\partial B_{\rho}\left(\boldsymbol{x}_{0}\right)}\left(|\nabla \boldsymbol{m}|^{2}+\Phi(\boldsymbol{m})\right) \mathrm{d} \mathcal{H}^{2} .
$$

Suppose that $\tilde{u} \in H^{2}\left(\Omega_{L}\right)$ is the solution of 3 for $\tilde{\boldsymbol{m}}$ instead of $\boldsymbol{m}$. Then

$$
\begin{aligned}
\int_{\Omega_{L}}|\nabla u-\nabla \tilde{u}|^{2} \mathrm{~d} \boldsymbol{x} & =\int_{\Omega_{L}}(\nabla u-\nabla \tilde{u}) \cdot(\boldsymbol{m}-\tilde{\boldsymbol{m}}) \mathrm{d} \boldsymbol{x} \\
& \leqslant\left(\frac{16 \pi}{3} \rho^{3} \int_{\Omega_{L}}|\nabla u-\nabla \tilde{u}|^{2} \mathrm{~d} \boldsymbol{x}\right)^{1 / 2} .
\end{aligned}
$$


Thus

$$
\int_{\Omega_{L}}|\nabla u-\nabla \tilde{u}|^{2} \mathrm{~d} x \leqslant \frac{16 \pi}{3} \rho^{3}
$$

and

$$
\int_{\Omega_{L}}\left(|\nabla \tilde{u}|^{2}-|\nabla u|^{2}\right) \mathrm{d} \boldsymbol{x}=\int_{\Omega_{L}}\left(|\nabla u-\nabla \tilde{u}|^{2}+2(\nabla \tilde{u}-\nabla u) \cdot \nabla u\right) \mathrm{d} \boldsymbol{x} \leqslant C_{2} \rho^{3 / 2}
$$

for a universal constant $C_{2}$. Since $\mathcal{J}_{L}\left(\boldsymbol{m} ; \boldsymbol{m}^{*}, u^{*}\right) \leqslant \mathcal{J}_{L}\left(\tilde{\boldsymbol{m}} ; \boldsymbol{m}^{*}, u^{*}\right)$, we conclude that

$$
\begin{aligned}
\int_{B_{\rho}\left(\boldsymbol{x}_{0}\right)}\left(|\nabla \boldsymbol{m}|^{2}+\Phi(\boldsymbol{m})\right) \mathrm{d} \boldsymbol{x} & \leqslant \int_{B_{\rho}\left(\boldsymbol{x}_{0}\right)}\left(|\nabla \tilde{\boldsymbol{m}}|^{2}+\Phi(\tilde{\boldsymbol{m}})\right) \mathrm{d} \boldsymbol{x}+\int_{\Omega_{L}}\left(|\nabla \tilde{u}|^{2}-|\nabla u|^{2}\right) \mathrm{d} \boldsymbol{x} \\
& \leqslant \rho \int_{\partial B_{\rho}\left(\boldsymbol{x}_{0}\right)}\left(|\nabla \boldsymbol{m}|^{2}+\Phi(\boldsymbol{m})\right) \mathrm{d} \mathcal{H}^{2}+C_{2} \rho^{3 / 2} .
\end{aligned}
$$

Therefore

$$
\frac{d}{d \rho}\left(\frac{1}{\rho} \int_{B_{\rho}\left(\boldsymbol{x}_{0}\right)}\left(|\nabla \boldsymbol{m}|^{2}+\Phi(\boldsymbol{m})\right) \mathrm{d} \boldsymbol{x}\right) \geqslant-\frac{C_{2}}{\sqrt{\rho}}
$$

and an integration over $[r, R]$ yields

$$
\frac{1}{r} \int_{B_{r}\left(\boldsymbol{x}_{0}\right)}\left(|\nabla \boldsymbol{m}|^{2}+\Phi(\boldsymbol{m})\right) \mathrm{d} \boldsymbol{x} \leqslant \frac{1}{R} \int_{B_{R}\left(\boldsymbol{x}_{0}\right)}\left(|\nabla \boldsymbol{m}|^{2}+\Phi(\boldsymbol{m})\right) \mathrm{d} \boldsymbol{x}+2 C_{2} \sqrt{R} .
$$

Similar arguments work if $\boldsymbol{x}_{0}$ is on $\partial Q_{1}(\mathbf{0}) \times(-L, L)$, provided that $B_{R}\left(\boldsymbol{x}_{0}\right) \cap \Omega_{L}$ is either exactly half of a ball or exactly a quarter of a ball. We conclude that there exists a universal constant $C_{3}$ such that

$$
\frac{1}{r} \int_{B_{r}\left(\boldsymbol{x}_{0}\right) \cap \Omega_{L}}\left(|\nabla \boldsymbol{m}|^{2}+\Phi(\boldsymbol{m})\right) \mathrm{d} \boldsymbol{x} \leqslant \frac{C_{3}}{R} \int_{B_{R}\left(\boldsymbol{x}_{0}\right) \cap \Omega_{L}}\left(|\nabla \boldsymbol{m}|^{2}+\Phi(\boldsymbol{m})\right) \mathrm{d} \boldsymbol{x}+C_{3} \sqrt{R}
$$

for all $\boldsymbol{x}_{0} \in Q_{1}^{\prime}(\mathbf{0}) \times(s-3 / 2, s+3 / 2)$ and $0<r \leqslant R \leqslant 1 / 2$. Choosing $R=\min \left\{\kappa^{2} / C_{3}^{2}, 1 / 2\right\}$, we now derive (4) for $r \leqslant R$. For $r>R$, the inequality is clear, as $C_{1}$ may depend on $\kappa$.

In the second step, we use the Euler-Lagrange equation for minimizers of $\mathcal{J}_{L}\left(\cdot ; \boldsymbol{m}^{*}, u^{*}\right)$. In order to calculate it, let $\phi \in C_{0}^{\infty}\left(\mathbb{R}^{2} \times(-L, L)\right)$ and define

$$
\boldsymbol{m}_{t}=\frac{\boldsymbol{m}+t \phi}{|\boldsymbol{m}+t \phi|}
$$

Moreover, let $u_{t} \in H^{2}\left(\Omega_{L}\right)$ be the solution of 3 ) for $\boldsymbol{m}_{t}$ instead of $\boldsymbol{m}$. Then

$$
\int_{\Omega_{L}} \nabla u \cdot\left(\nabla u_{t}-\nabla u\right) \mathrm{d} \boldsymbol{x}=\int_{\Omega_{L}} \nabla u \cdot\left(\boldsymbol{m}_{t}-\boldsymbol{m}\right) \mathrm{d} \boldsymbol{x},
$$

hence

$$
\left.\frac{\mathrm{d}}{\mathrm{d} t}\right|_{t=0} \int_{\Omega_{L}}\left|\nabla u_{t}\right|^{2} \mathrm{~d} \boldsymbol{x}=\left.2 \int_{\Omega_{L}} \nabla u \cdot \nabla \frac{\partial u_{t}}{\partial t}\right|_{t=0} \mathrm{~d} \boldsymbol{x}=2 \int_{\Omega_{L}}(\nabla u \cdot \phi-(\nabla u \cdot \boldsymbol{m})(\boldsymbol{\phi} \cdot \boldsymbol{m})) \mathrm{d} \boldsymbol{x} .
$$


With the usual calculations, we find the derivatives of the other two terms of $\mathcal{J}_{L}\left(\boldsymbol{m}_{t} ; \boldsymbol{m}^{*}, u^{*}\right)$, and we conclude that $m$ satisfies

$$
\Delta m+|\nabla m|^{2} m=f-(m \cdot f) m \quad \text { in } \Omega_{L}
$$

for the vector field

$$
f=\frac{1}{2} \operatorname{grad} \Phi(\boldsymbol{m})+\nabla u
$$

We also have the boundary conditions

$$
\begin{array}{ll}
\frac{\partial \boldsymbol{m}}{\partial x_{1}}=\mathbf{0} & \text { on }\{-1,1\} \times(-1,1) \times(-L, L), \\
\frac{\partial \boldsymbol{m}}{\partial x_{2}}=\mathbf{0} & \text { on }(-1,1) \times\{-1,1\} \times(-L, L),
\end{array}
$$

and of course $\boldsymbol{m}=\boldsymbol{m}^{*}$ on $Q_{1}^{\prime}(\mathbf{0}) \times\{-L, L\}$. An even extension of $\boldsymbol{m}$ across the faces of $\partial Q_{1}^{\prime}(\mathbf{0}) \times$ $(-L, L)$ satisfies an equation similar to (5). Since we have (4), Theorem 1 in [14] implies $\boldsymbol{m} \in$ $C^{0}\left(\overline{Q_{1}(\mathbf{0}, s)}, S^{2}\right)$ and

$$
\underset{Q_{1}(\mathbf{0}, s)}{\operatorname{osc}} \boldsymbol{m} \leqslant C_{4}\left(\int_{Q_{1}^{\prime}(\mathbf{0}) \times(s-2, s+2)}|\boldsymbol{f}|^{2} \mathrm{~d} \boldsymbol{x}+C_{1} \lambda+\kappa\right)^{1 / 2}
$$

for a universal constant $C_{4}$, provided that $\kappa$ and $\lambda$ are sufficiently small. We have

$$
|\boldsymbol{f}|^{2} \leqslant 2 \Phi(\boldsymbol{m})+2|\nabla u|^{2} .
$$

(Here we have used the special choice of $\Phi$. A different function will give rise to other constants.) Thus

$$
\underset{Q_{1}(\mathbf{0}, s)}{\operatorname{osc}} m \leqslant C_{4} \sqrt{\left(C_{1}+2\right) \lambda+\kappa}
$$

if $\kappa$ and $\lambda$ are small enough. This immediately gives the desired result.

Lemma 3.2 For any $L_{0} \geqslant 1$ there exists a constant $C$, depending only on $L_{0}$, such that the following is true. Let $L \in\left[1, L_{0}\right]$ and suppose that $m \in H^{1}\left(\Omega_{L}, S^{2}\right)$ and $u \in H^{2}\left(\Omega_{L}\right)$ with $\Delta u=\operatorname{div} \boldsymbol{m}$ in $\Omega_{L}$. If $\left|m_{3}-H_{3}\right| \leqslant \tau$ on $Q_{1}^{\prime}(\mathbf{0}) \times\{-L\}$, then

$$
\int_{\Omega_{L}} G_{\tau}(\boldsymbol{m}) \mathrm{d} \boldsymbol{x} \leqslant \theta \int_{\Omega_{L}}\left|\nabla m_{3}\right|^{2} \mathrm{~d} \boldsymbol{x}+C \int_{\Omega_{L}}\left|\nabla^{\prime} \boldsymbol{m}\right|^{2} \mathrm{~d} \boldsymbol{x}+\frac{C}{\theta} \int_{\Omega_{L}}|\nabla u|^{2} \mathrm{~d} \boldsymbol{x}
$$

for any $\theta \in(0,1]$.

Proof. Define

$$
\xi(\boldsymbol{x})= \begin{cases}m_{3}(\boldsymbol{x})-H_{3}-\tau & \text { if } m_{3}(\boldsymbol{x})>H_{3}+\tau, \\ 0 & \text { if } H_{3}-\tau \leqslant m_{3}(\boldsymbol{x}) \leqslant H_{3}+\tau, \\ m_{3}(\boldsymbol{x})-H_{3}+\tau & \text { if } m_{3}(\boldsymbol{x})<H_{3}-\tau,\end{cases}
$$

so that $G_{\tau}(\boldsymbol{m})=\xi^{2}$. Let $\eta \in C_{0}^{\infty}\left(Q_{1}^{\prime}(\mathbf{0})\right)$ with

$$
\int_{Q_{1}^{\prime}(\mathbf{0})} \eta^{2} \mathrm{~d} \boldsymbol{x}^{\prime}=4
$$


For $s \in(-L, L)$, set

$$
\zeta(s)=\frac{1}{2} \int_{Q_{1}^{\prime}(\mathbf{0})} \eta^{2}\left(\boldsymbol{x}^{\prime}\right) \xi^{2}\left(\boldsymbol{x}^{\prime}, s\right) \mathrm{d} \boldsymbol{x}^{\prime} .
$$

Then $\zeta(-L)=0$. For almost every $s \in(-L, L)$, we have

$$
\begin{aligned}
\zeta^{\prime}(s) & =\int_{Q_{1}^{\prime}(\mathbf{0})} \eta^{2}\left(\boldsymbol{x}^{\prime}\right) \xi\left(\boldsymbol{x}^{\prime}, s\right) \frac{\partial m_{3}}{\partial x_{3}}\left(\boldsymbol{x}^{\prime}, s\right) \mathrm{d} \boldsymbol{x}^{\prime} \\
& =\int_{Q_{1}^{\prime}(\mathbf{0})} \eta^{2}\left(\boldsymbol{x}^{\prime}\right) \xi\left(\boldsymbol{x}^{\prime}, s\right)\left(\Delta u\left(\boldsymbol{x}^{\prime}, s\right)-\operatorname{div}^{\prime} \boldsymbol{m}^{\prime}\left(\boldsymbol{x}^{\prime}, s\right)\right) \mathrm{d} \boldsymbol{x}^{\prime}
\end{aligned}
$$

where

$$
\operatorname{div}^{\prime} \boldsymbol{m}^{\prime}=\frac{\partial m_{1}}{\partial x_{1}}+\frac{\partial m_{2}}{\partial x_{2}}
$$

Thus for almost all $x_{3} \in(-L, L)$, we find that

$$
\zeta\left(x_{3}\right)=\int_{-L}^{x_{3}} \int_{Q_{1}^{\prime}(\mathbf{0})} \eta^{2}\left(\boldsymbol{x}^{\prime}\right) \xi\left(\boldsymbol{x}^{\prime}, s\right)\left(\Delta u\left(\boldsymbol{x}^{\prime}, s\right)-\operatorname{div}^{\prime} \boldsymbol{m}^{\prime}\left(\boldsymbol{x}^{\prime}, s\right)\right) \mathrm{d} \boldsymbol{x}^{\prime} \mathrm{d} s .
$$

We compute

$$
\begin{aligned}
& \int_{-L}^{x_{3}} \int_{Q_{1}^{\prime}(\mathbf{0})} \eta^{2}\left(\boldsymbol{x}^{\prime}\right) \xi\left(\boldsymbol{x}^{\prime}, s\right) \Delta u\left(\boldsymbol{x}^{\prime}, s\right) \mathrm{d} \boldsymbol{x}^{\prime} \mathrm{d} s \\
&=\int_{Q_{1}^{\prime}(\mathbf{0})} \eta^{2}\left(\boldsymbol{x}^{\prime}\right) \xi\left(\boldsymbol{x}^{\prime}, x_{3}\right) \frac{\partial u}{\partial x_{3}}\left(\boldsymbol{x}^{\prime}, x_{3}\right) \mathrm{d} \boldsymbol{x}^{\prime} \\
&-\int_{-L}^{x_{3}} \int_{Q_{1}^{\prime}(\mathbf{0})} \eta^{2}\left(\boldsymbol{x}^{\prime}\right) \nabla \xi\left(\boldsymbol{x}^{\prime}, s\right) \cdot \nabla u\left(\boldsymbol{x}^{\prime}, s\right) \mathrm{d} \boldsymbol{x}^{\prime} \mathrm{d} s \\
&-2 \int_{-L}^{x_{3}} \int_{Q_{1}^{\prime}(\mathbf{0})} \eta\left(\boldsymbol{x}^{\prime}\right) \xi\left(\boldsymbol{x}^{\prime}, s\right) \nabla^{\prime} \eta\left(\boldsymbol{x}^{\prime}\right) \cdot \nabla^{\prime} u\left(\boldsymbol{x}^{\prime}, s\right) \mathrm{d} \boldsymbol{x}^{\prime} \mathrm{d} s .
\end{aligned}
$$

Hence there is a constant $C_{1}=C_{1}(\eta)$ such that for all $\theta_{1}, \theta_{2}>0$,

$$
\begin{aligned}
\zeta\left(x_{3}\right) \leqslant & C_{1} \theta_{1} \zeta\left(x_{3}\right)+\frac{C_{1}}{\theta_{1}} \int_{Q_{1}^{\prime}(\mathbf{0})}\left|\nabla u\left(\boldsymbol{x}^{\prime}, x_{3}\right)\right|^{2} \mathrm{~d} \boldsymbol{x}^{\prime}+C_{1} \theta_{2} \int_{\Omega_{L}}\left|\nabla m_{3}\right|^{2} \mathrm{~d} \boldsymbol{x} \\
& +C_{1} \theta_{1} \int_{-L}^{L} \zeta(s) \mathrm{d} s+C_{1}\left(\frac{1}{\theta_{1}}+\frac{1}{\theta_{2}}\right) \int_{\Omega_{L}}|\nabla u|^{2} \mathrm{~d} \boldsymbol{x}+\frac{C_{1}}{\theta_{1}} \int_{\Omega_{L}}\left|\boldsymbol{\nabla}^{\prime} \boldsymbol{m}\right|^{2} \mathrm{~d} \boldsymbol{x} .
\end{aligned}
$$

Integrating over $(-L, L)$, we see that

$$
\begin{aligned}
\int_{-L}^{L} \zeta\left(x_{3}\right) \mathrm{d} x_{3} \leqslant & C_{2} \theta_{1} \int_{-L}^{L} \zeta\left(x_{3}\right) \mathrm{d} x_{3}+C_{2} \theta_{2} \int_{\Omega_{L}}\left|\nabla m_{3}\right|^{2} \mathrm{~d} \boldsymbol{x} \\
& +C_{2}\left(\frac{1}{\theta_{1}}+\frac{1}{\theta_{2}}\right) \int_{\Omega_{L}}|\nabla u|^{2} \mathrm{~d} \boldsymbol{x}+\frac{C_{2}}{\theta_{1}} \int_{\Omega_{L}}\left|\nabla^{\prime} \boldsymbol{m}\right|^{2} \mathrm{~d} \boldsymbol{x}
\end{aligned}
$$


for a constant $C_{2}$ that depends on $\eta$ and $L_{0}$. If we choose $\theta_{1}$ small enough, then it follows that there exists a constant $C_{3}=C_{3}\left(\eta, L_{0}\right)$ with

$$
\int_{-L}^{L} \zeta\left(x_{3}\right) \mathrm{d} x_{3} \leqslant C_{3} \theta_{2} \int_{\Omega_{L}}\left|\nabla m_{3}\right|^{2} \mathrm{~d} \boldsymbol{x}+\frac{C_{3}}{\theta_{2}} \int_{\Omega_{L}}|\nabla u|^{2} \mathrm{~d} \boldsymbol{x}+C_{3} \int_{\Omega_{L}}\left|\nabla^{\prime} \boldsymbol{m}\right|^{2} \mathrm{~d} \boldsymbol{x}
$$

for any $\theta_{2} \in(0,1]$.

With the help of the Poincaré inequality, we see that there exists a constant $C_{4}$, dependent only on $\eta$, such that

$$
\int_{Q_{1}^{\prime}(\mathbf{0})} \xi^{2}\left(\boldsymbol{x}^{\prime}, x_{3}\right) \mathrm{d} \boldsymbol{x}^{\prime} \leqslant 4 \zeta\left(x_{3}\right)+C_{4} \int_{Q_{1}^{\prime}(\mathbf{0})}\left|\boldsymbol{\nabla}^{\prime} m_{3}\left(\boldsymbol{x}^{\prime}, x_{3}\right)\right|^{2} \mathrm{~d} \boldsymbol{x}^{\prime}
$$

for almost every $x_{3} \in(-L, L)$. Therefore we find

$$
\int_{\Omega_{L}} G_{\tau}(\boldsymbol{m}) \mathrm{d} \boldsymbol{x} \leqslant 4 C_{3} \theta_{2} \int_{\Omega_{L}}\left|\nabla m_{3}\right|^{2} \mathrm{~d} \boldsymbol{x}+\frac{4 C_{3}}{\theta_{2}} \int_{\Omega_{L}}|\nabla u|^{2} \mathrm{~d} \boldsymbol{x}+\left(4 C_{3}+C_{4}\right) \int_{\Omega_{L}}\left|\nabla^{\prime} \boldsymbol{m}\right|^{2} \mathrm{~d} \boldsymbol{x}
$$

The desired inequality follows immediately.

REMARK It is clear that the inequality of this lemma can be proved with the same arguments if the condition $\left|m_{3}-H_{3}\right| \leqslant \tau$ holds on $Q_{1}^{\prime}(\mathbf{0}) \times\{L\}$ instead of $Q_{1}^{\prime}(0) \times\{-L\}$.

Lemma 3.3 There exist two constants $c$ and $L_{0} \geqslant 2$, dependent only on $\tau$, with the following property. Let $L \geqslant L_{0}$ and suppose that $m \in H^{1}\left(\Omega_{L}, S^{2}\right)$ and $u \in H^{2}\left(\Omega_{L}\right)$ with $\Delta u=\operatorname{div} \boldsymbol{m}$ in $\Omega_{L}$. Then for any $\theta \in(0,1]$,

$$
\int_{\Omega_{L}}\left(|\nabla \boldsymbol{m}|^{2}+\Phi(\boldsymbol{m})+|\nabla u|^{2}\right) \mathrm{d} \boldsymbol{x} \geqslant \int_{Q_{1}^{\prime}(\mathbf{0})} \delta_{\left(1-c \theta^{2}\right)\left(\Phi+\theta G_{\tau}\right)}\left(\boldsymbol{m}\left(\boldsymbol{x}^{\prime},-L\right), \boldsymbol{m}\left(\boldsymbol{x}^{\prime}, L\right)\right) \mathrm{d} \boldsymbol{x}^{\prime} .
$$

Proof. First note that we may assume without loss of generality that $\boldsymbol{m}$ minimizes $\mathcal{J}_{L}(\cdot ; \boldsymbol{m}, u)$ in $\mathcal{A}_{L}(\boldsymbol{m})$. If it does not, we simply replace $\boldsymbol{m}$ by a minimizer and $u$ by the solution of the corresponding boundary value problem. The left-hand side of the inequality in the lemma does not become larger thereby, and the right-hand side does not change at all. Moreover, we may assume that

$$
\int_{\Omega_{L}}\left(|\nabla \boldsymbol{m}|^{2}+\Phi(\boldsymbol{m})+|\nabla u|^{2}\right) \mathrm{d} \boldsymbol{x} \leqslant 4
$$

for the assertion is trivial otherwise.

Let $s \in[2-L, L-2]$ and suppose that $\Lambda$ is the constant from Lemma 3.1 Then we have either

$$
\int_{Q_{1}^{\prime}(\mathbf{0}) \times(s-2, s+2)}\left(|\nabla \boldsymbol{m}|^{2}+\Phi(\boldsymbol{m})+|\nabla u|^{2}\right) \mathrm{d} \boldsymbol{x} \geqslant \Lambda
$$

or $\boldsymbol{m} \in C^{0}\left(\overline{Q_{1}(\mathbf{0}, s)}, S^{2}\right)$ and

$$
\underset{Q_{1}(\mathbf{0}, s)}{\mathrm{osc}} m \leqslant \tau / 2 .
$$

If the latter is true and if there exists a point $\boldsymbol{x}_{0} \in Q_{1}(\mathbf{0}, s)$ with $\left|m_{3}\left(\boldsymbol{x}_{0}\right)-H_{3}\right| \geqslant \tau$, it follows that

$$
\int_{Q_{1}(\mathbf{0}, s)} \Phi(\boldsymbol{m}) \mathrm{d} \boldsymbol{x} \geqslant 2 \tau^{2}
$$


Hence there exists a number $K>0$, dependent only on $\tau$, such that we always have either

$$
\int_{Q_{1}^{\prime}(\mathbf{0}) \times(s-2, s+2)}\left(|\nabla \boldsymbol{m}|^{2}+\Phi(\boldsymbol{m})+|\nabla u|^{2}\right) \mathrm{d} \boldsymbol{x} \geqslant K
$$

or

$$
G_{\tau}(\boldsymbol{m})=0 \quad \text { in } \overline{Q_{1}(\mathbf{0}, s)} .
$$

We conclude that there exist certain numbers $s_{1}, \ldots, s_{I} \in[1-L, L-1]$ such that $G_{\tau}(\boldsymbol{m})=0$ in $\Omega_{L} \backslash \bigcup_{i=1}^{I} Q_{1}\left(\mathbf{0}, s_{i}\right)$, and the number $I$ is bounded by a constant $I_{0}$ that depends only on $\tau$. We can then construct another set of numbers $\sigma_{1}, \ldots, \sigma_{J}$ with $J \leqslant I_{0}$ and a number $R \in\left[1,4^{I_{0}}\right]$ such that

- $R-L \leqslant \sigma_{j} \leqslant L-R$ for $j=1, \ldots, J$,

- $\left|\sigma_{i}-\sigma_{j}\right| \geqslant 4 R$ for $i \neq j$, and

- $G_{\tau}(\boldsymbol{m})=0$ in $\Omega_{L} \backslash\left(Q_{1}^{\prime}(\mathbf{0}) \times \bigcup_{j=1}^{J}\left(\sigma_{j}-R, \sigma_{j}+R\right)\right)$.

If $L>2 R$, then we can apply Lemma 3.2 (or the remark following its proof) to the restriction of $\boldsymbol{m}$ to $Q_{1}^{\prime}(\mathbf{0}) \times\left(\sigma_{j}-R, \sigma_{j}+R\right)$ for every $j=1, \ldots, J$. This gives a constant $C_{1}=C_{1}(\tau) \geqslant 1$ such that

$$
\int_{\Omega_{L}} G_{\tau}(\boldsymbol{m}) \mathrm{d} \boldsymbol{x} \leqslant \int_{\Omega_{L}}\left(\theta\left|\nabla m_{3}\right|^{2}+C_{1}\left|\nabla^{\prime} \boldsymbol{m}\right|^{2}+\frac{C_{1}}{\theta}|\nabla u|^{2}\right) \mathrm{d} \boldsymbol{x}
$$

for any $\theta \in(0,1]$. For $\kappa=1 / 2 C_{1}$ we then find

$$
\int_{\Omega_{L}}\left(\left(1-\kappa \theta^{2}\right)\left|\frac{\partial \boldsymbol{m}}{\partial x_{3}}\right|^{2}+\Phi(\boldsymbol{m})+\kappa \theta G_{\tau}(\boldsymbol{m})\right) \mathrm{d} \boldsymbol{x} \leqslant \int_{\Omega_{L}}\left(|\nabla \boldsymbol{m}|^{2}+\Phi(\boldsymbol{m})+|\nabla u|^{2}\right) \mathrm{d} \boldsymbol{x} .
$$

We know that the function

$$
\phi\left(\boldsymbol{x}^{\prime}, x_{3}\right)=\delta_{\left(1-\kappa \theta^{2}\right)\left(\Phi+\kappa \theta G_{\tau}\right)}\left(\boldsymbol{m}\left(\boldsymbol{x}^{\prime}, x_{3}\right), \boldsymbol{m}\left(\boldsymbol{x}^{\prime},-L\right)\right)
$$

satisfies

$$
\left|\frac{\partial \phi}{\partial x_{3}}\right| \leqslant\left(1-\kappa \theta^{2}\right)\left|\frac{\partial \boldsymbol{m}}{\partial x_{3}}\right|^{2}+\Phi(\boldsymbol{m})+\kappa \theta G_{\tau}(\boldsymbol{m}) .
$$

Thus

$$
\int_{Q_{1}^{\prime}(\mathbf{0})} \phi\left(\boldsymbol{x}^{\prime}, L\right) \mathrm{d} \boldsymbol{x}^{\prime} \leqslant \int_{\Omega_{L}}\left(|\boldsymbol{\nabla} \boldsymbol{m}|^{2}+\Phi(\boldsymbol{m})+|\nabla u|^{2}\right) \mathrm{d} \boldsymbol{x} .
$$

The claim of the lemma now follows for $c=\kappa^{-2}$.

Proof of Proposition 3.1. Define $\tilde{\boldsymbol{m}}(\boldsymbol{x})=\boldsymbol{m}(\epsilon \boldsymbol{x})$ and $\tilde{u}(\boldsymbol{x})=(1 / \epsilon) u(\epsilon \boldsymbol{x})$. Then for $L=1 / \epsilon$, we have

$$
\int_{Q_{1}(\mathbf{0})}\left(\epsilon|\nabla \boldsymbol{m}|^{2}+\frac{1}{\epsilon}\left(\Phi(\boldsymbol{m})+|\nabla u|^{2}\right)\right) \mathrm{d} \boldsymbol{x}=\epsilon^{2} \int_{Q_{L}(\mathbf{0})}\left(|\nabla \tilde{\boldsymbol{m}}|^{2}+\Phi(\tilde{\boldsymbol{m}})+|\nabla \tilde{u}|^{2}\right) \mathrm{d} \boldsymbol{x}
$$

and

$$
\begin{aligned}
\int_{Q_{1}^{\prime}(\mathbf{0})} \delta_{\left(1-c \theta^{2}\right)\left(\Phi+\theta G_{\tau}\right)}\left(\boldsymbol{m}\left(x^{\prime},-1\right),\right. & \left.\boldsymbol{m}\left(\boldsymbol{x}^{\prime}, 1\right)\right) \mathrm{d} \boldsymbol{x}^{\prime} \\
= & \epsilon^{2} \int_{Q_{L}^{\prime}(\mathbf{0})} \delta_{\left(1-c \theta^{2}\right)\left(\Phi+\theta G_{\tau}\right)}\left(\boldsymbol{m}\left(x^{\prime},-L\right), \boldsymbol{m}\left(\boldsymbol{x}^{\prime}, L\right)\right) \mathrm{d} \boldsymbol{x}^{\prime} .
\end{aligned}
$$


We assume for simplicity that $\epsilon=2^{-\ell}$ for some $\ell \in \mathbb{N}$. Then we can decompose $Q_{L}(\mathbf{0})$ into cuboids of the form $Q_{1}^{\prime}\left(x_{0}^{\prime}\right) \times(-L, L)$, and the desired inequality follows directly from Lemma 3.3 .

\section{Proofs of the main results}

The results of the previous section are stated in a form that is useful when we study domain walls parallel to $\mathbb{R}^{2} \times\{0\}$. In general, we have to replace the function $G_{\tau}$ with

$$
G_{\tau}^{\boldsymbol{\nu}}(\boldsymbol{y})=\left(\max \left\{\left|\left(\boldsymbol{y}-\boldsymbol{a}_{+}\right) \cdot \boldsymbol{\nu}\right|-\tau, 0\right\}\right)^{2}
$$

for a unit vector $\boldsymbol{\nu}$ (perpendicular to the domain wall). If $\boldsymbol{\nu} \in N$, then a change of coordinates takes us back to the previous situation, and we can use Proposition 3.1 to estimate the energy density. But in order to obtain a statement as in Theorem 2.1, we need another lemma.

Lemma 4.1 Let $\tau, c>0$ and $\boldsymbol{\nu} \in S^{2}$. Either there exists a path $\gamma \in \Gamma\left(\boldsymbol{a}_{+}, \boldsymbol{a}_{-}\right)$such that $\left|\left(\gamma-\boldsymbol{a}_{+}\right) \cdot \boldsymbol{\nu}\right| \leqslant \tau$ in $[0,1]$ and

$$
\mathcal{D}(\Phi)=2 \int_{0}^{1} \sqrt{\Phi(\gamma)}\left|\gamma^{\prime}\right| \mathrm{d} t
$$

or there exists a number $\theta_{0}>0$ such that

$$
\mathcal{D}(\Phi)<\mathcal{D}\left(\left(1-c \theta^{2}\right)\left(\Phi+\theta G_{\tau}^{\nu}\right)\right)
$$

for all $\theta \in\left(0, \theta_{0}\right]$.

REMARK Equation (6) implies that $\gamma$ is a shortest geodesic for $g_{\Phi}$ connecting $\boldsymbol{a}_{+}$and $\boldsymbol{a}_{-}$. For the function $\Phi$ used in this paper, this means that $\gamma$ describes a circular arc in the plane spanned by $\boldsymbol{H}$ and $\boldsymbol{a}_{+}-\boldsymbol{a}_{-}$. If $c(\tau)$ is the number from Proposition 3.1 belonging to $\tau$, then it follows that the function

$$
W(\boldsymbol{\nu})=\sup _{\tau>0} \sup _{0<\theta \leqslant 1} \mathcal{D}\left(\left(1-c(\tau) \theta^{2}\right)\left(\Phi+\theta G_{\tau}^{\boldsymbol{\nu}}\right)\right), \quad \boldsymbol{\nu} \in N,
$$

satisfies $W(\boldsymbol{\nu})>\mathcal{D}(\Phi)$ unless $\boldsymbol{\nu}$ is perpendicular to $\boldsymbol{H}$.

Proof. For $\theta \in(0,1]$ define the function

$$
\Phi_{\theta}=\left(1-c \theta^{2}\right)\left(\Phi+\theta G_{\tau}^{\boldsymbol{\nu}}\right)
$$

on $S^{2}$. We assume that there exists a sequence $\theta_{k} \searrow 0$ such that

$$
\mathcal{D}(\Phi) \geqslant \mathcal{D}\left(\Phi_{\theta_{k}}\right),
$$

and we have to show that there exists a path $\gamma \in \Gamma\left(\boldsymbol{a}_{+}, \boldsymbol{a}_{-}\right)$with $G_{\tau}^{\nu} \circ \gamma=0$ that satisfies (6).

For each $k$, choose $\gamma_{k} \in \Gamma\left(\boldsymbol{a}_{+}, \boldsymbol{a}_{-}\right)$with

$$
2 \int_{0}^{1} \sqrt{\Phi_{\theta_{k}}\left(\gamma_{k}(t)\right)}\left|\gamma_{k}^{\prime}(t)\right| \mathrm{d} t \leqslant \mathcal{D}\left(\Phi_{\theta_{k}}\right)+\frac{\theta_{k}}{k}
$$

and such that $\left|\gamma_{k}^{\prime}\right|$ is constant for each $k$, say $\left|\gamma_{k}^{\prime}(t)\right| \equiv b_{k}$. We may assume that $b_{k} \rightarrow b>0$ as $k \rightarrow \infty$ (after the choice of a subsequence if necessary). By the theorem of Arzelà-Ascoli, we may 
further assume that there exists a path $\gamma \in \Gamma\left(\boldsymbol{a}_{+}, \boldsymbol{a}_{-}\right)$such that $\gamma_{k} \rightarrow \gamma$ uniformly. Then clearly $\gamma$ satisfies (6).

Fix $t \in[0,1]$ such that $\gamma(t) \notin\left\{\boldsymbol{a}_{+}, \boldsymbol{a}_{-}\right\}$. Then $\gamma_{k}(t) \notin\left\{\boldsymbol{a}_{+}, \boldsymbol{a}_{-}\right\}$for any sufficiently large $k$. Fix such a $k$ for the moment and consider the function

$$
f(\theta)=\sqrt{\Phi_{\theta}\left(\gamma_{k}(t)\right)} .
$$

By the mean value theorem, there exists a number $\tilde{\theta}_{k} \in\left(0, \theta_{k}\right)$ such that

$$
\frac{f\left(\theta_{k}\right)-f(0)}{\theta_{k}}=f^{\prime}\left(\tilde{\theta}_{k}\right) \text {. }
$$

That is,

$$
\frac{\sqrt{\Phi_{\theta_{k}}\left(\gamma_{k}(t)\right)}-\sqrt{\Phi\left(\gamma_{k}(t)\right)}}{\theta_{k}}=\frac{\left(1-3 c \tilde{\theta}_{k}^{2}\right) G_{\tau}^{\nu}\left(\gamma_{k}(t)\right)-2 c \tilde{\theta}_{k} \Phi\left(\gamma_{k}(t)\right)}{2 \sqrt{\Phi_{\tilde{\theta}_{k}}\left(\gamma_{k}(t)\right)}}
$$

It follows that

$$
\lim _{k \rightarrow \infty} \frac{\sqrt{\Phi_{\theta_{k}}\left(\gamma_{k}(t)\right)}-\sqrt{\Phi\left(\gamma_{k}(t)\right)}}{\theta_{k}}=\frac{G_{\tau}^{\nu}(\gamma(t))}{2 \sqrt{\Phi(\gamma(t))}}
$$

Define

$$
\zeta(t)=\frac{G_{\tau}^{\nu}(\gamma(t))}{2 \sqrt{\Phi(\gamma(t))}}
$$

if $\gamma(t) \notin\left\{\boldsymbol{a}_{+}, \boldsymbol{a}_{-}\right\}$and $\zeta(t)=0$ else. Then Fatou's lemma implies

$$
\begin{aligned}
b \int_{0}^{1} \zeta(t) \mathrm{d} t & \leqslant \liminf _{k \rightarrow \infty} \frac{b_{k}}{\theta_{k}} \int_{0}^{1}\left(\sqrt{\Phi_{\theta_{k}}\left(\gamma_{k}(t)\right)}-\sqrt{\Phi\left(\gamma_{k}(t)\right)}\right) \mathrm{d} t \\
& \leqslant \liminf _{k \rightarrow \infty} \frac{1}{\theta_{k}}\left(\mathcal{D}\left(\Phi_{\theta_{k}}\right)+\frac{\theta_{k}}{k}-\mathcal{D}(\Phi)\right) \leqslant 0
\end{aligned}
$$

That is, we have $G_{\tau}^{\nu}(\gamma(t))=0$ for every $t \in[0,1]$. Therefore, the path $\gamma$ has the required properties.

Proof of Theorem 2.1 Suppose that $\boldsymbol{m}_{\epsilon} \in H^{1}\left(\Omega, S^{2}\right)$ and $u_{\epsilon} \in H^{2}(\Omega)$ satisfy the hypotheses of Theorem 2.1, that is, $\Delta u_{\epsilon}=\operatorname{div} \boldsymbol{m}_{\epsilon}$ in $\Omega$ and

$$
\underset{\epsilon \searrow 0}{\liminf } \int_{\Omega}\left(\epsilon\left|\nabla \boldsymbol{m}_{\epsilon}\right|^{2}+\frac{1}{\epsilon}\left(\Phi\left(\boldsymbol{m}_{\epsilon}\right)+\left|\nabla u_{\epsilon}\right|^{2}\right)\right) \mathrm{d} \boldsymbol{x}<\infty .
$$

Define the functions

$$
\phi_{\epsilon}=\delta_{\Phi}\left(\boldsymbol{m}_{\epsilon}, \boldsymbol{a}_{-}\right)
$$

on $\Omega$. Moreover, consider the measures $\mu_{\epsilon}$ on $\Omega$ defined by the condition that

$$
\int_{\Omega} \eta \mathrm{d} \mu_{\epsilon}=\int_{\Omega} \eta\left(\epsilon\left|\nabla \boldsymbol{m}_{\epsilon}\right|^{2}+\frac{1}{\epsilon}\left(\Phi\left(\boldsymbol{m}_{\epsilon}\right)+\left|\nabla u_{\epsilon}\right|^{2}\right)\right) \mathrm{d} \boldsymbol{x}
$$

for $\eta \in C_{0}^{0}(\Omega)$. We have

$$
\int_{\Omega} \eta\left|\nabla \phi_{\epsilon}\right| \mathrm{d} x \leqslant \int_{\Omega} \eta \mathrm{d} \mu_{\epsilon}
$$


if $\eta \geqslant 0$ and

$$
\liminf _{\epsilon \searrow 0} \mu_{\epsilon}(\Omega)<\infty
$$

Thus there exist

- a sequence $\epsilon_{k} \searrow 0$,

- a function $\phi \in \mathrm{BV}(\Omega)$ with $\phi(x) \in\{0, \mathcal{D}(\Phi)\}$ almost everywhere, and

- a Radon measure $\mu$ on $\Omega$

such that $\phi_{\epsilon_{k}} \rightarrow \phi$ in $L^{1}(\Omega)$ as well as pointwise almost everywhere and $\mu_{\epsilon_{k}} \rightarrow \mu$ weakly* in the dual space of $C_{0}^{0}(\Omega)$. Moreover, we have $|\boldsymbol{D} \phi| \leqslant \mu$. Define

$$
\Omega_{+}=\{\boldsymbol{x} \in \Omega: \phi(\boldsymbol{x})=\mathcal{D}(\Phi)\} \quad \text { and } \quad \Omega_{-}=\Omega \backslash \Omega_{+} .
$$

These are sets of finite perimeter. Setting

$$
\boldsymbol{m}(\boldsymbol{x})= \begin{cases}\boldsymbol{a}_{+} & \text {if } \boldsymbol{x} \in \Omega_{+}, \\ \boldsymbol{a}_{-} & \text {if } \boldsymbol{x} \in \Omega_{-},\end{cases}
$$

we clearly have $\boldsymbol{m}_{\epsilon_{k}}(\boldsymbol{x}) \rightarrow \boldsymbol{m}(\boldsymbol{x})$ almost everywhere. Moreover, any $\eta \in C_{0}^{\infty}(\Omega)$ satisfies

$$
\int_{\Omega} \nabla \eta \cdot \boldsymbol{m} \mathrm{d} \boldsymbol{x}=\lim _{k \rightarrow \infty} \int_{\Omega} \nabla \eta \cdot \boldsymbol{m}_{\epsilon_{k}} \mathrm{~d} \boldsymbol{x}=\lim _{k \rightarrow \infty} \int_{\Omega} \nabla \eta \cdot \nabla u_{\epsilon_{k}} \mathrm{~d} \boldsymbol{x}=0 .
$$

That is, we have $\operatorname{div} \boldsymbol{m}=0$, which means that $\boldsymbol{\nu}_{\Omega_{+}}(\boldsymbol{x}) \in N$ for $\mathcal{H}^{2}$-almost every $\boldsymbol{x} \in \mathcal{F} \Omega_{+}$.

For every $\boldsymbol{x} \in \mathcal{F} \Omega_{+}$, choose a cube $\tilde{Q}(\boldsymbol{x})$ of side length 1 centred at $\mathbf{0}$ such that $\boldsymbol{\nu}_{\Omega_{+}}(\boldsymbol{x})$ is perpendicular to two of its faces and let $\tilde{Q}_{r}(\boldsymbol{x})=r \tilde{Q}(\mathbf{0})+\boldsymbol{x}$. Define

$$
\Theta(\boldsymbol{x})=\limsup _{r \searrow 0} \frac{\mu\left(\tilde{Q}_{r}(\boldsymbol{x})\right)}{4 r^{2}} .
$$

Fix a point $\boldsymbol{x}_{0} \in \mathcal{F} \Omega_{+}$and let $\tau>0$ and $\theta \in(0,1]$. Let $c(\tau)$ be the constant from Proposition 3.1 that belongs to $\tau$. We want to show that

$$
\Theta\left(\boldsymbol{x}_{0}\right) \geqslant \mathcal{D}\left(\left(1-c(\tau) \theta^{2}\right)\left(\Phi+\theta G_{\tau}^{\boldsymbol{\nu}_{\Omega_{+}}\left(\boldsymbol{x}_{0}\right)}\right)\right)
$$

Then we have $\Theta\left(\boldsymbol{x}_{0}\right) \geqslant W\left(\boldsymbol{\nu}_{\Omega_{+}}\left(\boldsymbol{x}_{0}\right)\right)$ for the function $W$ defined by (7). Standard arguments then show that $W$ satisfies inequality (2), which means that it has the properties stated in Theorem 2.1. Thus the proof is complete once we have verified (8).

For this purpose we use a blow-up argument. For simplicity we assume that $\nu_{\Omega_{+}}\left(\boldsymbol{x}_{0}\right)=(0,0,1)$ and $\tilde{Q}_{r}\left(\boldsymbol{x}_{0}\right)=Q_{r}\left(\boldsymbol{x}_{0}\right)$. That is,

$$
\Theta\left(\boldsymbol{x}_{0}\right)=\limsup _{r \searrow 0} \frac{\mu\left(Q_{r}\left(\boldsymbol{x}_{0}\right)\right)}{4 r^{2}} .
$$

We choose a sequence $r_{k} \searrow 0$. Then we can replace $\left\{\epsilon_{k}\right\}$ by a subsequence such that for every $k$ we have

$$
\frac{\epsilon_{k}}{r_{k}}+\frac{1}{r_{k}^{3}} \int_{Q_{r_{k}}\left(\boldsymbol{x}_{0}\right)}\left|\phi_{\epsilon_{k}}-\phi\right| \mathrm{d} \boldsymbol{x} \leqslant \frac{1}{k}
$$


and

$$
\frac{1}{r_{k}^{2}} \int_{Q_{r_{k}}\left(\boldsymbol{x}_{0}\right)}\left(\epsilon_{k}\left|\nabla \boldsymbol{m}_{\epsilon_{k}}\right|^{2}+\frac{1}{\epsilon_{k}}\left(\Phi\left(\boldsymbol{m}_{\epsilon_{k}}\right)+\left|\nabla u_{\epsilon_{k}}\right|^{2}\right)\right) \mathrm{d} \boldsymbol{x} \leqslant \frac{\mu\left(Q_{r_{k}}\left(\boldsymbol{x}_{0}\right)\right)}{r_{k}^{2}}+\frac{1}{k} .
$$

Set $\tilde{\epsilon}_{k}=\epsilon_{k} / r_{k}$ and consider the rescaled maps

$$
\tilde{\boldsymbol{m}}_{k}(\boldsymbol{x})=\boldsymbol{m}_{\epsilon_{k}}\left(r_{k} \boldsymbol{x}+\boldsymbol{x}_{0}\right)
$$

and the functions

$$
\tilde{u}_{k}=\frac{1}{r_{k}} u_{\epsilon_{k}}\left(r_{k} \boldsymbol{x}+\boldsymbol{x}_{0}\right) .
$$

Then we have

- $\tilde{\epsilon}_{k} \rightarrow 0$ as $k \rightarrow \infty$,

- $\tilde{\boldsymbol{m}}_{k}(\boldsymbol{x}) \rightarrow \boldsymbol{a}_{-}$almost everywhere on $Q_{1}^{\prime}(\mathbf{0}) \times\{-1\}$,

- $\tilde{\boldsymbol{m}}_{k}(\boldsymbol{x}) \rightarrow \boldsymbol{a}_{+}$almost everywhere on $Q_{1}^{\prime}(\mathbf{0}) \times\{1\}$,

and furthermore,

$$
\Theta\left(\boldsymbol{x}_{0}\right) \geqslant \frac{1}{4} \limsup _{k \rightarrow \infty} \int_{Q_{1}(\mathbf{0})}\left(\tilde{\epsilon}_{k}\left|\nabla \tilde{\boldsymbol{m}}_{k}\right|^{2}+\frac{1}{\tilde{\epsilon}_{k}}\left(\Phi\left(\tilde{\boldsymbol{m}}_{k}\right)+\left|\nabla \tilde{u}_{k}\right|^{2}\right)\right) \mathrm{d} \boldsymbol{x} .
$$

Proposition 3.1 finally implies 8 .

Proof of Proposition 2.1 Remember that we assume $\Omega=(-1,1)^{3}$ and $\boldsymbol{\nu}=(0,0,1)$. Moreover, we have $H_{2}=0$ and $0<H_{3} \leqslant \beta$ for a number $\beta>0$ that remains to be determined.

Let $\gamma \in \Gamma\left(\boldsymbol{a}_{-}, \boldsymbol{a}_{+}\right)$be a shortest geodesic for the Riemannian metric $g_{\Phi+F^{\nu}}$ connecting $\boldsymbol{a}_{-}$ and $\boldsymbol{a}_{+}$, parametrized in a way such that $\gamma^{\prime}(t) \neq 0$ for all $t \in(0,1)$. If $\beta$ is sufficiently small, then $\gamma$ does not follow the circular arc in the plane $\mathbb{R} \times\{0\} \times \mathbb{R}$ (because a semicircle in the plane $\mathbb{R}^{2} \times\left\{H_{3}\right\}$ is shorter with respect to this metric). It is then clear that $\gamma_{3}(t)>H_{3}$ and $\gamma_{2}(t) \neq 0$ for all $t \in(0,1)$. In particular, $\gamma_{2}(t)\left(\gamma_{3}(t)-H_{3}\right)$ does not change its sign.

For $\omega \in(-\pi / 2, \pi / 2)$, let $\boldsymbol{\nu}_{\omega}=(0, \sin \omega, \cos \omega)$ and $\Phi_{\omega}=\Phi+F^{\boldsymbol{\nu}_{\omega}}$. Consider the function

$$
f(\omega)=2 \int_{0}^{1} \sqrt{\Phi_{\omega}(\gamma(t))}\left|\gamma^{\prime}(t)\right| \mathrm{d} t .
$$

We compute

$$
f^{\prime}(0)=2 \int_{0}^{1} \frac{\gamma_{2}(t)\left(\gamma_{3}(t)-H_{3}\right)}{\sqrt{\Phi_{\omega}(\gamma(t))}}\left|\gamma^{\prime}(t)\right| \mathrm{d} t \neq 0 .
$$

Therefore

$$
\left.\frac{\mathrm{d}}{\mathrm{d} \omega}\right|_{\omega=0} \frac{f(\omega)}{\cos \omega} \neq 0,
$$

and there exists an angle $\omega \in(-\pi / 2, \pi / 2)$ such that

$$
\frac{f(\omega)}{\cos \omega}<f(0)=\mathcal{D}\left(\Phi+F^{\nu}\right) .
$$

We fix $\omega$ with this property. 
We need to reparametrize $\gamma$ suitably. To this end, let $k \in \mathbb{N}$ and consider the function

$$
\zeta_{k}(t)=\int_{0}^{t} \frac{\left|\gamma^{\prime}(s)\right|}{\sqrt{\Phi_{\omega}(\gamma(s))+k^{-2}}} \mathrm{~d} s .
$$

Obviously $\zeta_{k}$ is an increasing function. Let $T_{k}=\frac{1}{2} \zeta_{k}(1)$ and note that

$$
T_{k} \leqslant \frac{k}{2} \int_{0}^{1}\left|\gamma^{\prime}(s)\right| \mathrm{d} s .
$$

Consider the inverse function $\zeta_{k}^{-1}$ on $\left[0,2 T_{k}\right]$. The curve

$$
c_{k}(s)=\gamma\left(\zeta_{k}^{-1}\left(s+T_{k}\right)\right), \quad-T_{k} \leqslant s \leqslant T_{k},
$$

satisfies $\boldsymbol{c}\left(-T_{k}\right)=\boldsymbol{a}_{-}$and $\boldsymbol{c}\left(T_{k}\right)=\boldsymbol{a}_{+}$. Moreover,

$$
\left|\boldsymbol{c}_{k}^{\prime}(s)\right|=\sqrt{\Phi_{\omega}\left(\boldsymbol{c}_{k}(s)\right)+k^{-2}} .
$$

Hence the numbers

$$
\alpha_{k}=\int_{-T_{k}}^{T_{k}}\left(\left|\boldsymbol{c}_{k}^{\prime}\right|^{2}+\Phi_{\omega}\left(\boldsymbol{c}_{k}\right)\right) \mathrm{d} s
$$

satisfy

$$
\alpha_{k}-f(\omega)=\int_{-T_{k}}^{T_{k}}\left(2 \Phi_{\omega}\left(\boldsymbol{c}_{k}\right)+\frac{1}{k^{2}}-2 \sqrt{\Phi_{\omega}\left(\boldsymbol{c}_{k}\right)\left(\Phi_{\omega}\left(\boldsymbol{c}_{k}\right)+k^{-2}\right)}\right) \mathrm{d} s \leqslant \frac{2 T_{k}}{k^{2}} .
$$

Because of (9), this implies

$$
\limsup _{k \rightarrow \infty} \alpha_{k} \leqslant f(\omega) .
$$

We extend $\boldsymbol{c}_{k}$ to $\mathbb{R}$ by $\boldsymbol{c}_{k}(s)=\boldsymbol{a}_{-}$for $s<-T_{k}$ and $\boldsymbol{c}_{k}(s)=\boldsymbol{a}_{+}$for $s>T_{k}$. Moreover, we define

$$
v_{k}(s)=\int_{0}^{s} \boldsymbol{\nu}_{\omega} \cdot\left(\boldsymbol{c}_{k}(t)-\boldsymbol{a}_{+}\right) \mathrm{d} t, \quad s \in \mathbb{R} .
$$

Note that $v_{k}$ is constant in $\left(-\infty,-T_{k}\right]$ and in $\left[T_{k}, \infty\right)$.

Let $\Omega_{k}=(-1,1) \times(-1 / k, 1 / k) \times(-1,1)$. For $\epsilon>0$ and $\boldsymbol{x} \in \Omega_{k}$, define

$$
\tilde{\boldsymbol{m}}_{k}^{\epsilon}(\boldsymbol{x})=\boldsymbol{c}_{k}\left(\frac{\boldsymbol{x} \cdot \boldsymbol{\nu}_{\omega}}{\epsilon}\right) \quad \text { and } \quad \tilde{u}_{k}^{\epsilon}(\boldsymbol{x})=\epsilon v_{k}\left(\frac{\boldsymbol{x} \cdot \boldsymbol{\nu}_{\omega}}{\epsilon}\right) .
$$

Then we have $\Delta \tilde{u}_{k}^{\epsilon}=\operatorname{div} \tilde{\boldsymbol{m}}_{k}^{\epsilon}$ in $\Omega_{k}$. Moreover, we compute

$$
\left|\nabla \tilde{u}_{k}^{\epsilon}\right|^{2}=\left(\boldsymbol{\nu}_{\omega} \cdot\left(\tilde{\boldsymbol{m}}_{k}^{\epsilon}-\boldsymbol{a}_{+}\right)\right)^{2}=F^{\boldsymbol{\nu}_{\omega}}\left(\tilde{\boldsymbol{m}}_{k}^{\epsilon}\right)
$$

and therefore by 10$]$ and the definition of $\Phi_{\omega}$, we have

$$
\lim _{\epsilon \searrow 0} \int_{\Omega_{k}}\left(\epsilon\left|\nabla \tilde{\boldsymbol{m}}_{k}^{\epsilon}\right|^{2}+\frac{1}{\epsilon}\left(\Phi\left(\tilde{\boldsymbol{m}}_{k}^{\epsilon}\right)+\left|\nabla \tilde{u}_{k}^{\epsilon}\right|^{2}\right)\right) \mathrm{d} \boldsymbol{x}=\frac{4 \alpha_{k}}{k \cos \omega} .
$$


Next we define a family of maps $\boldsymbol{m}_{k}^{\epsilon} \in H^{1}\left(\Omega_{k}, S^{2}\right)$ and a family of functions $\hat{u}_{k}^{\epsilon} \in H^{2}\left(\Omega_{k}\right)$ as follows. For $\epsilon T_{k}-1 / k<x_{2}<1 / k-\epsilon T_{k}$ we set $\boldsymbol{m}_{k}^{\epsilon}(\boldsymbol{x})=\tilde{\boldsymbol{m}}_{k}^{\epsilon}(\boldsymbol{x})$ and $\hat{u}_{k}^{\epsilon}(\boldsymbol{x})=\tilde{u}_{k}^{\epsilon}(\boldsymbol{x})$. Otherwise, if $\boldsymbol{x} \cdot \boldsymbol{\nu}_{\omega}<-\epsilon T_{k}$, then $\boldsymbol{m}_{k}^{\epsilon}(\boldsymbol{x})=\boldsymbol{a}_{-}$and $\hat{u}_{k}^{\epsilon}(\boldsymbol{x})=\epsilon v_{k}\left(-T_{k}\right)$, and if $\boldsymbol{x} \cdot \boldsymbol{\nu}_{\omega}>\epsilon T_{k}$, then $\boldsymbol{m}_{k}^{\epsilon}(\boldsymbol{x})=\boldsymbol{a}_{+}$and $\hat{u}_{k}^{\epsilon}(\boldsymbol{x})=\epsilon v_{k}\left(T_{k}\right)$. In the remaining part of $\Omega_{k}$, we define $\boldsymbol{m}_{k}^{\epsilon}$ and $\hat{u}_{k}^{\epsilon}$ in a way such that

$$
\boldsymbol{m}_{k}^{\epsilon}\left(x_{1}, \pm k^{-1}, x_{3}\right) \in \mathbb{R} \times\{0\} \times \mathbb{R} \quad \text { and } \quad \frac{\partial \hat{u}_{k}^{\epsilon}}{\partial x_{2}}\left(x_{1}, \pm k^{-1}, x_{3}\right)=0,
$$

and furthermore,

$$
\limsup _{\epsilon \searrow 0} \epsilon\left(\left\|\nabla \boldsymbol{m}_{k}^{\epsilon}\right\|_{L^{\infty}\left(\Omega_{k}\right)}+\left\|\Delta \hat{u}_{k}^{\epsilon}\right\|_{L^{\infty}\left(\Omega_{k}\right)}\right)<\infty .
$$

Let $\boldsymbol{R}: S^{2} \rightarrow S^{2}$ be the reflection at $\mathbb{R} \times\{0\} \times \mathbb{R}$, that is, $\boldsymbol{R}\left(y_{1}, y_{2}, y_{3}\right)=\left(y_{1},-y_{2}, y_{3}\right)$. We now extend $\boldsymbol{m}_{k}^{\epsilon}$ to $\Omega$ by

$$
\boldsymbol{m}_{k}^{\epsilon}\left(x_{1}, x_{2}+2 \ell / k, x_{3}\right)= \begin{cases}\boldsymbol{m}_{k}^{\epsilon}\left(x_{1}, x_{2}, x_{3}\right) & \text { if } \ell \text { is even } \\ \boldsymbol{R}\left(\boldsymbol{m}_{k}^{\epsilon}\left(x_{1},-x_{2}, x_{3}\right)\right) & \text { if } \ell \text { is odd }\end{cases}
$$

Similarly, we extend $\hat{u}_{k}^{\epsilon}$ to $\Omega$ by

$$
\hat{u}_{k}^{\epsilon}\left(x_{1}, x_{2}+2 \ell / k, x_{3}\right)= \begin{cases}\hat{u}_{k}^{\epsilon}\left(x_{1}, x_{2}, x_{3}\right) & \text { if } \ell \text { is even } \\ \hat{u}_{k}^{\epsilon}\left(x_{1},-x_{2}, x_{3}\right) & \text { if } \ell \text { is odd. }\end{cases}
$$

Then there exist certain sets $U_{k}^{\epsilon} \subset \Omega$ such that $\Delta \hat{u}_{k}^{\epsilon}=\operatorname{div} \boldsymbol{m}_{k}^{\epsilon}$ outside of $U_{k}^{\epsilon}$ and

$$
\limsup _{\epsilon \searrow 0} \frac{\left|U_{k}^{\epsilon}\right|}{\epsilon^{2}}<\infty
$$

Because of (11), it follows that

$$
\frac{1}{\epsilon}\left\|\Delta \hat{u}_{h}^{\epsilon}-\operatorname{div} \boldsymbol{m}_{k}^{\epsilon}\right\|_{L^{p}(\Omega)}^{2} \rightarrow 0 \quad \text { as } \epsilon \searrow 0
$$

for every $p<4 / 3$. Let $u_{k}^{\epsilon} \in H^{2}(\Omega)$ be the solutions of the boundary value problems

$$
\begin{array}{ccrl}
\Delta u_{k}^{\epsilon} & =\operatorname{div} \boldsymbol{m}_{k}^{\epsilon} & & \text { in } \Omega, \\
u_{k}^{\epsilon}=\hat{u}_{k}^{\epsilon} & & \text { on } \partial \Omega .
\end{array}
$$

Then we have

$$
\frac{1}{\epsilon} \int_{\Omega}\left|\nabla u_{k}^{\epsilon}-\nabla \hat{u}_{k}^{\epsilon}\right|^{2} \mathrm{~d} \boldsymbol{x} \leqslant \frac{1}{\epsilon}\left\|\Delta \hat{u}_{h}^{\epsilon}-\operatorname{div} \boldsymbol{m}_{k}^{\epsilon}\right\|_{L^{6 / 5}(\Omega)}^{2} \rightarrow 0 \quad \text { as } \epsilon \searrow 0 .
$$

Therefore,

and

$$
\lim _{\epsilon \searrow 0} \int_{\Omega}\left(\epsilon\left|\nabla \boldsymbol{m}_{k}^{\epsilon}\right|^{2}+\frac{1}{\epsilon}\left(\Phi\left(\boldsymbol{m}_{k}^{\epsilon}\right)+\left|\nabla u_{k}^{\epsilon}\right|^{2}\right)\right) \mathrm{d} \boldsymbol{x}=\frac{4 \alpha_{k}}{\cos \omega}
$$

$$
\limsup _{k \rightarrow \infty} \frac{\alpha_{k}}{\cos \omega} \leqslant \frac{f(\omega)}{\cos \omega}<\mathcal{D}\left(\Phi+F^{\nu}\right)
$$

Finally, by the construction of $\boldsymbol{m}_{k}^{\epsilon}$, we have a sequence of maps $\boldsymbol{m}_{k}^{0}: \Omega \rightarrow\left\{\boldsymbol{a}_{-}, \boldsymbol{a}_{+}\right\}$such that $\boldsymbol{m}_{k}^{\epsilon} \rightarrow \boldsymbol{m}_{k}^{0}$ in $L^{1}\left(\Omega, \mathbb{R}^{3}\right)$ as $\epsilon \searrow 0$. Let $\boldsymbol{m}(\boldsymbol{x})=\boldsymbol{a}_{-}$for $x_{3}<0$ and $\boldsymbol{m}(\boldsymbol{x})=\boldsymbol{a}_{+}$for $x_{3}>0$. Then we also have $\boldsymbol{m}_{k}^{0} \rightarrow \boldsymbol{m}$ in $L^{1}\left(\Omega, \mathbb{R}^{3}\right)$. To conclude the proof, it now suffices to choose a suitable diagonal sequence of $\left\{\boldsymbol{m}_{k}^{\epsilon}\right\}$ and extract a subsequence if necessary. 


\section{REFERENCES}

1. Alouges, F., Rivière, T., \& Serfaty, S. Néel and cross-tie wall energies for planar micromagnetic configurations. ESAIM Control Optim. Calc. Var. 8 (2002), 31-68. Zbl 1092.82047 MR 1932944

2. Ambrosio, L., Fusco, N., \& Pallara, D. Functions of Bounded Variation and Free Discontinuity Problems. Oxford Math. Monogr., Oxford Univ. Press, New York (2000). Zbl 0957.49001 MR 1857292

3. Anzellotti, G., Baldo, S., \& Visintin, A. Asymptotic behavior of the Landau-Lifshitz model of ferromagnetism. Appl. Math. Optim. 23 (1991), 171-192. Zbl 0746.49032 MR 1086467

4. BALdo, S. Minimal interface criterion for phase transitions in mixtures of Cahn-Hilliard fluids. Ann. Inst. H. Poincaré Anal. Non Linéaire 7 (1990), 67-90. Zbl $0702.49009 \mid$ MR 1051228

5. Bertotti, G. Hysteresis in Magnetism: For Physicists, Material Scientists, and Engineers. Academic Press, San Diego (1998).

6. Choкsi, R., \& Konn, R.V. Bounds on the micromagnetic energy of a uniaxial ferromagnet. Comm. Pure Appl. Math. 51 (1998), 259-289. Zbl 0909.49004 MR 1488515

7. Chокsi, R., Kонn, R. V., \& Отто, F. Domain branching in uniaxial ferromagnets: a scaling law for the minimum energy. Comm. Math. Phys. 201 (1999), 61-79. Zbl 1023.82011 MR 1669433

8. De Lellis, C., \& Otтo, F. Structure of entropy solutions to the eikonal equation. J. Eur. Math. Soc. 5 (2003), 107-145. Zbl 1053.49028 MR 1985613

9. Fonseca, I., \& TARTAR, L. The gradient theory of phase transitions for systems with two potential wells. Proc. Roy. Soc. Edinburgh Sect. A 111 (1989), 89-102. Zbl 0676.49005 MR 0985992

10. Hubert, A., \& SCHÄFER, R. Magnetic Domains. Springer, Berlin (1998).

11. Jabin, P.-E., Otto, F., \& Perthame, B. Line-energy Ginzburg-Landau models: zero-energy states. Ann. Scuola Norm. Sup. Pisa Cl. Sci. (5) 1 (2002), 187-202. Zbl 1072.35051 MR 1994807

12. ModicA, L. The gradient theory of phase transitions and the minimal interface criterion. Arch. Ration. Mech. Anal. 98 (1987), 123-142. Zbl 0616.76004 MR 0866718

13. Modica, L., \& Mortola, S. Un esempio di $\Gamma^{-}$-convergenza. Boll. Un. Mat. Ital. B (5) 14 (1977), 285-299. Zbl 0356.49008 MR 0445362

14. Moser, R. Regularity for the approximated harmonic map equation and application to the heat flow for harmonic maps. Math. Z. 243 (2003), 263-289. Zbl 1027.58014 MR 1961867

15. RiviÈre, T., \& SERfATY, S. Limiting domain wall energy for a problem related to micromagnetics. Comm. Pure Appl. Math. 54 (2001), 294-338. Zbl 1031.35142 MR 1809740

16. Rivière, T., \& SERfaty, S. Compactness, kinetic formulation, and entropies for a problem related to micromagnetics. Comm. Partial Differential Equations 28 (2003), 249-269. Zbl 1094.35125 MR 1974456

17. Sternberg, P. The effect of a singular perturbation on nonconvex variational problems. Arch. Ration. Mech. Anal. 101 (1988), 209-260. Zbl 0647.49021||MR 0930124 